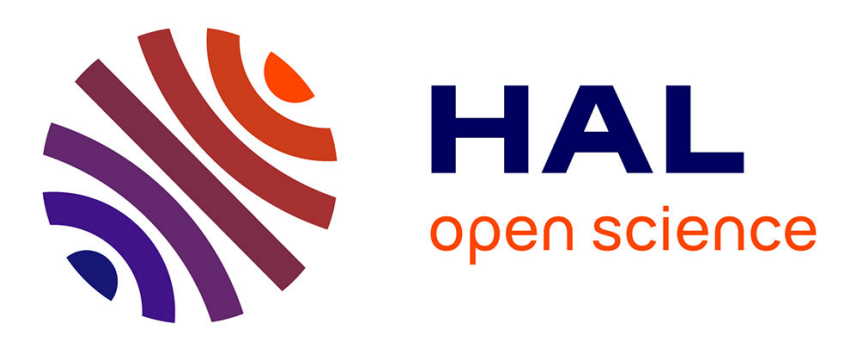

\title{
Uncertainties in conditional probability tables of discrete Bayesian Belief Networks: A comprehensive review
}

\author{
Jeremy Rohmer
}

\section{To cite this version:}

Jeremy Rohmer. Uncertainties in conditional probability tables of discrete Bayesian Belief Networks: A comprehensive review. Engineering Applications of Artificial Intelligence, 2020, 88, 10.1016/j.engappai.2019.103384 . hal-02386579

\section{HAL Id: hal-02386579 https://hal-brgm.archives-ouvertes.fr/hal-02386579}

Submitted on 29 Nov 2019

HAL is a multi-disciplinary open access archive for the deposit and dissemination of scientific research documents, whether they are published or not. The documents may come from teaching and research institutions in France or abroad, or from public or private research centers.
L'archive ouverte pluridisciplinaire HAL, est destinée au dépôt et à la diffusion de documents scientifiques de niveau recherche, publiés ou non, émanant des établissements d'enseignement et de recherche français ou étrangers, des laboratoires publics ou privés. 
1 Uncertainties in conditional probability tables of discrete

2 Bayesian Belief Networks: a comprehensive review

\section{Abstract \\ Abstract}

\section{Jeremy Rohmer ${ }^{1}$}

[1]\{BRGM, 3 av. C. Guillemin - 45060 Orléans Cedex 2 - France\}

Correspondence to: J. Rohmer (j.rohmer@brgm.fr)

Discrete Bayesian Belief Network (BBN) has become a popular method for the analysis of complex systems in various domains of application. One of its pillar is the specification of the parameters of the probabilistic dependence model (i.e. the cause-effect relation) represented via a Conditional Probability Table (CPT). Depending on the available data (observations, prior knowledge, expert-based information, etc.), CPTs can be populated in different manners, i.e. different assumptions can be made and different methods are available, which might lead to uncertain BBN-based results. Through an extensive review study of the past ten years, we aim at addressing three questions related to the CPT uncertainties. First, we show how to constrain these uncertainties either using elicitation of expert inputs, or using a combination of scarce data and expert-derived information. Second, we show how to integrate these uncertainties in the $\mathrm{BBN}$-based analysis through propagation procedures either using probabilities or imprecise probabilities within the setting of credal or evidential networks. Finally, we show how to test the robustness of the BBN-based results to these uncertainties via sensitivity analysis specifically dedicated to BBNs. A special care was paid to describe the best practices for the implementation of the reviewed methods and the remaining gaps.

Keywords: Bayesian Belief Network; Conditional Probability Table; Expert Elicitation; Sensitivity Analysis; Credal Network; Evidential Network. 


\section{Introduction}

Bayesian Belief Network (BBN) has become an increasingly popular method for the analysis of complex systems in various domains of application, like ecosystems (Milns et al., 2010), genetics and biology (Scutari et al., 2014), agriculture (Drury et al., 2017), industry (Weber et al., 2012), finance forecasting (Malagrino et al., 2018), marine safety (Hänninen et al., 2014), human reliability assessment (Mkrtchyan et al., 2015), nuclear power plants (Kwag and Gupta, 2017), aviation risk analysis (Brooker, 2011), coastal systems (Jäger et al., 2018), structure reliability assessments (Langseth and Portinale, 2007), multi-hazard risk assessments (Gehl and D’Ayala, 2016), etc.

Its benefits are: (1) its high flexibility to model any causal relationships; (2) its capability to integrate information from any kind of sources, including experimental data, historical data, and prior expert opinion, and (3) its capability to answer probabilistic queries about them and to find out updated knowledge of the state of a subset of variables when other variables (i.e. the evidence variables) are observed.

Formally, a Bayesian belief Network (BBN) is a class of graphical model (see Jensen, 2001 for a complete and detailed introduction to BBNs), which allows to synthetically represent relations among random variables by means of a directed acyclic graph (DAG) composed of nodes (i.e. the states of the random variables) and arcs (i.e. dependency between nodes). The value of the nodes may be discrete or continuous, and we focus here on the former case, which is the most widely used. For instance, a Boolean node representing the state of a system component can be either "True" or "False". The nodes connected by an arc are called the parent nodes and child nodes respectively. One child node may have several parent nodes, meaning that this node is affected by several factors. Similarly, a parent node could have several child nodes, meaning that this factor may have influences on several other factors. Conditional probabilities are the probabilities that reflect the degree of influence of the parent nodes on the child node. For BBNs with discrete nodes, the probabilistic dependence (i.e. the cause-effect relation) is often represented via a table called a Conditional Probability Table (CPT).

As an illustration, Fig. 1 depicts the binary BBN adapted by van der Gaag et al. (2013) from Cooper (1984) in the field of oncology. The network is composed of 6 nodes and 6 arcs. Node MC refers to metastatic cancer, which may potentially lead to the development of a brain tumor (node B) and may give rise to an increased level of serum calcium (node ISC). The presence of a brain tumour can be established from a CT scan (node CT). Another indicator of the presence 
level of serum calcium are both likely to cause a patient to fall into a coma (the node $\mathrm{C}$ is connected to node B and node ISC). The conditional probabilistic relationships between the nodes (CPT entries) are provided in Fig. 1 next to the corresponding nodes. For instance, the probability that a patient falls into coma given brain tumor and increased level of serum calcium corresponds to the first entry of the table ( $1^{\text {st }}$ row, $1^{\text {st }}$ column), namely $\mathrm{P}(\mathrm{C}=$ True $\mid \mathrm{C}=$ True, $\mathrm{ISC}=$ True $)=0.80$.

(6)

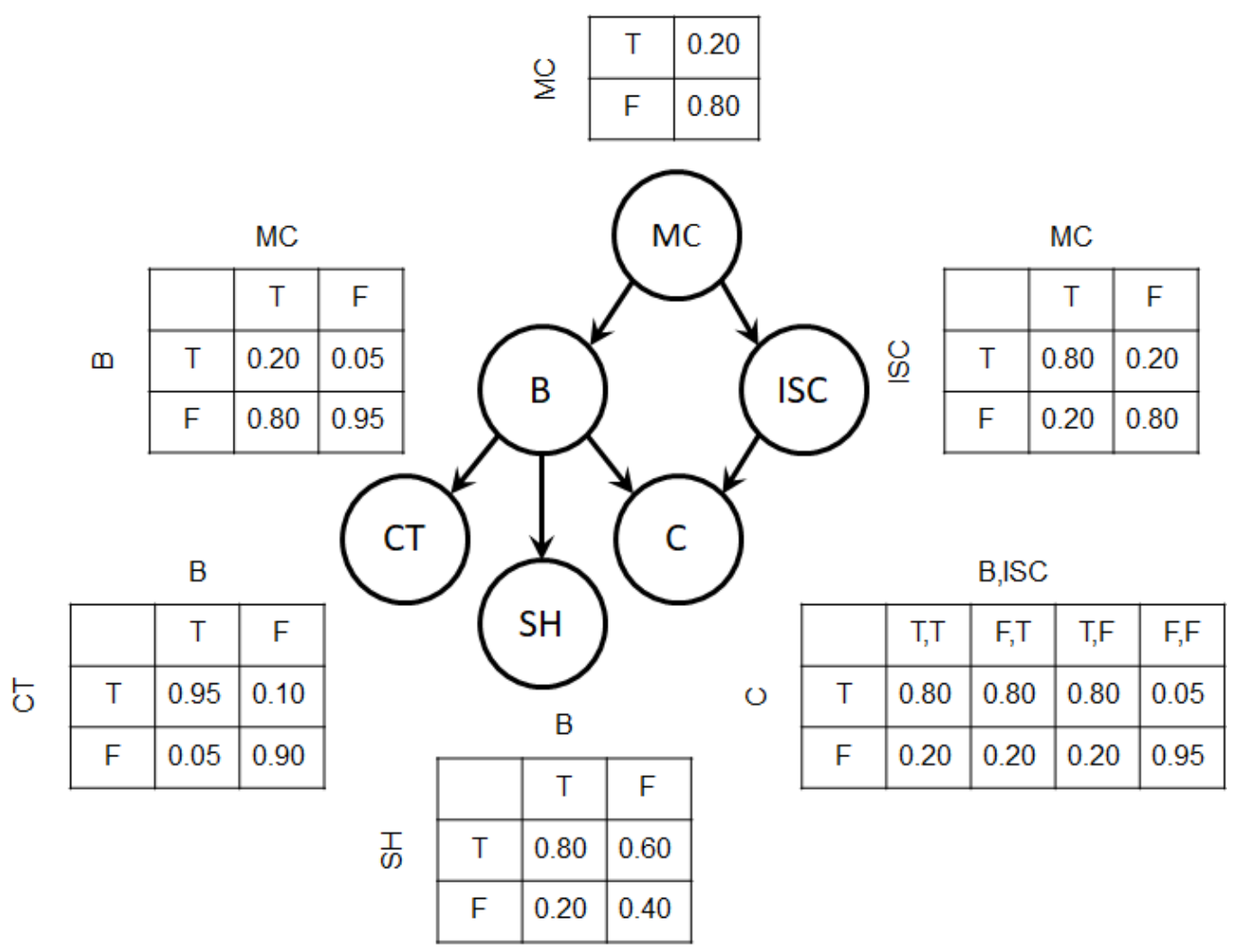

Figure 1. Binary BBN adapted by van der Gaag et al. (2013) from Cooper (1984) in the field of oncology. The tables (called CPT) next to the nodes provide the conditional probabilities values.

[Figure 1 about here]

Two key ingredients are necessary to build a BBN, namely (1) the graph structure with the direction of the arcs, i.e. the DAG; (2) the states of nodes and the strength of the relationships between nodes, i.e. the CPT. In the present study, we assume that the DAG model has already been determined and restrict the analysis to the quantification of the BBN relationships. The process of deriving the CPTs and its associated uncertainties is recognized in the literature as one of the most delicate part of the BBN development (e.g., Chen and Pollino, 2012; Druzdzel 
and van der Gaag, 2000; Marcot et al., 2006; Cain, 2001, etc.). It should, however, be noted that the process of DAG derivation (i.e. building the graph structure plus the directions; also known as causal structure learning) has its own challenges as well, in particular when the learning is based on data (see e.g., a comprehensive review by Heinze-Deml et al., 2018).

Depending on the available data (observations, prior knowledge, expert-based information, etc.), CPTs can be evaluated in different manners, i.e. different assumptions can be made and different methods are available leading to different BBN-based results, hence resulting in uncertain BBN-based results. This raises the following questions: (1) how to constrain the uncertainties related to CPT derivation, i.e. what are the methods that are available to minimize these uncertainties? (2) how to integrate these uncertainties in the BBN-based analysis, i.e. what are the methods for propagating these uncertainties? (3) how to test the robustness of the BBNbased results to these uncertainties, i.e. what are the methods for identifying the most influential uncertainties? These questions are addressed below through an extensive review of studies performed in the past ten years by focusing on discrete BBNs that can be used for modelling complex causal relationships, for merging different information sources, for prediction, and for belief/evidence propagation (i.e. probabilistic queries). Continuous BBNs (i.e. BBNs with continuous nodes) and dynamic BBNs (i.e. BBNs adapted to model systems evolving over time) are out of the scope of the review.

The paper is organized as follows. The first section describes more specifically the problem of populating the CPT parameters directly from data/observations. This first part highlights the necessity for overcoming the lack of data by complementing with additional sources of information. Sect. 3 explores an expert-based option for constraining the uncertainties related to data incompleteness, namely by completing with different expert-based sources of information. Sect. 4 provides an overview of the different approaches embedded in different uncertainty analysis settings for evaluating the impacts of CPT uncertainties, either using probabilities or imprecise probabilities. Sect. 5 further addresses the problem of screening these uncertainties by describing sensitivity analysis techniques. Finally, Sect. 6 summarizes the main findings and discusses the open questions.

\section{Learning CPT from data}

In this section, we address the issues related to deriving the CPT entries from data. Sect. 2.1 first discusses the problem of performing this task by using only data. Sect 2.2 and 2.3 further discuss two practical difficulties, namely: (1) the presence of missing values and (2) the problem of translating observations related to continuous variables into a limited number of 
113

discrete states. Finally, Sect. 2.4 describes methods that make the most out of scarce data while exploiting qualitative information provided by experts.

\subsection{A pure data-driven approach}

Let us consider a BBN composed of $\mathrm{n}$ discrete nodes $X_{\mathrm{i}=1, \ldots, \mathrm{n}}$. Let us denote $r_{\mathrm{i}}$ the cardinality of $X_{\mathrm{i}}$ and $q_{\mathrm{i}}$ the one of the parent set of $X_{\mathrm{i}}$, denoted $\mathrm{pa}\left(X_{\mathrm{i}}\right)$. The $\mathrm{k}^{\text {th }}$ probability value of the conditional probability distribution is $\theta_{\mathrm{ijk}}=P\left(X_{i}=k \mid p a\left(X_{i}\right)=j\right)$ where $\mathrm{i}=1, \ldots, \mathrm{n} ; \mathrm{j}=1, \ldots, q_{\mathrm{i}}$; $\mathrm{k}=1, \ldots, r_{\mathrm{i}}$.

In data rich contexts, CPT parameters can be evaluated by computing the appropriate frequencies from data. An example is provided by Chojnacki et al. (2019) for fire safety analysis where more than 1 million of numerical simulation results are used. This method corresponds to the maximum likelihood estimation (MLE), which is described below.

Let us consider a dataset $D$ where a total number $N_{\mathrm{ij}}$ of data records are available for which $\mathrm{pa}\left(X_{\mathrm{i}}\right)$ is in the state $\mathrm{j}$ and where $N_{\mathrm{ijk}}$ data records are available for which $X_{\mathrm{i}}$ is in the state $\mathrm{k}$ and $\operatorname{pa}\left(X_{\mathrm{i}}\right)$ is in the state j. MLE aims at maximizing the log-likelihood function $l($.) of $\theta$ given $D$ as follows

$l(\theta \mid D)=\log (P(D \mid \theta))=\sum_{\mathrm{ijk}} N_{\mathrm{ijk}} \log \left(\theta_{\mathrm{ijk}}\right)$

The solution is then $\frac{N_{\mathrm{ijk}}}{N_{\mathrm{ij}}}$.

The MLE method however fails to find good estimates due to data scarcity when $N_{\mathrm{ij}} \approx 0$, i.e. when training data are not sufficient in number in some specific variable state configurations. Examples of such contexts are not rare in practice; see e.g. rare disease diagnostic (Seixas et al., 2014), accident prevention (e.g., Hänninen, 2014), reliability analysis (e.g., Musharraf et al., 2014), etc. This problem is even worsened when the number of nodes increases. Recall that the number of conditional probabilities is exponential with the number of its parent nodes, i.e. for a node with i states and $\mathrm{k}$ parent nodes and if each parent node has $\mathrm{n}$ states, $(\mathrm{i}-1) \times \mathrm{n}^{\mathrm{k}} \mathrm{CPT}$ entry values have to be specified. For instance, a binary node with 2 binary parent nodes imposes to specify 4 entries, whereas for a ternary node with 2 ternary nodes, this number reaches 18 . 


\subsection{Dealing with missing values}

The process for parameter learning of discrete BBNs may be complicated in the presence of missing values. This can be handled by means of different algorithms. The most popular ones are Expectation Maximization (Dempster et al., 1977) and Gibbs sampling (Geman and Geman, 1984). Yet, they both assume that the values are missing at random. This hypothesis may not always be true in practice. Alternative methods have been proposed to overcome this disadvantage, like AI\&M procedure (Jaeger, 2006), the RBE algorithm (Ramoni and Sebastiani, 2001), and the maximum entropy method (Cowell, 1999). Other methods have also been developed to speed up the learning process, like generalized conjugate gradient algorithm by Thiesson (1995) or the online updating of rules (Bauer et al., 1997). To deal with both missing data and qualitative influences (as described in Sect. 2.4), some initiatives have been proposed like the one of Masegosa et al. (2016), who further improved the combined Isotonic Regression - EM approach.

\subsection{Discretising continuous variables}

A second practical difficulty for parameter learning of discrete BBNs is inherent to the main assumption introduced by discrete BBNs, namely that data should be represented by a limited numbers of outcomes. This imposes to discretize continuous variables. This process might, however, lead to a loss of information, and potentially to an increase of the associated computational effort, because the size of discrete BBNs increases approximately exponentially with the number of discrete states of its nodes. Nojavan et al. (2017) investigated the implications of several mathematical methods for constructing discrete distributions in an unsupervised manner. Using a simple 3-node BBN describing chlorophyll concentrations in Finnish lakes, the authors evaluated the impact on the developed BBNs of the number of intervals and of the choice of the type of discretization methods. Three techniques were investigated, namely in which the data are divided into groups: (1) of equal length; (2) of equal sample size; (3) for which the moments of the discretized distribution match with the moments of the continuous data. They showed that none of the models did uniformly well in all comparison criteria (sum of squared errors, accuracy, area under the receiving operating characteristic curve) for the considered case. They concluded that they cannot justify using one discretization method against others. Using a 4-node BBN from the domain of coastal erosion, Beuzen et al. (2018) extended the tests to other types of discretization methods, namely manual and supervised techniques. They showed, on their specific test case, that supervised methods 
$p(\theta \mid D) \propto P(D \mid \theta) P(D) \propto \prod_{i j k} \theta_{i j k}\left(\alpha_{i j k}+N_{i j k}\right)-1$

197 This equation results in the estimate of $\theta_{\mathrm{ijk}}$ as $\frac{N_{\mathrm{ijk}}+\alpha_{\mathrm{ijk}}-1}{N_{\mathrm{ij}}+\alpha_{\mathrm{ij}}-1}$, which combines information from

led to a $\mathrm{BBN}$ of the highest average predictive skill, followed by the one with manual discretization. They also outlined the advantages of the different methods, namely that:

- Manual methods allow ensuring physical meaningful BBNs;

- Supervised methods can autonomously and optimally discretize variables and may be preferred when predictive skill is a modelling priority;

- Unsupervised methods are computationally simple and versatile.

Depending on the objective, some specific discretization algorithms have also been developed; for instance, Zwirglmaier and Straub (2016) developed specific methods to deal with rare events in reliability analysis; Neil et al. (2007) proposed a dynamic discretization method to perform inference in hybrid BBNs, i.e. both dealing with continuous and discrete variables.

\subsection{Combining scarce data and expert judgements}

When data are scarce, the parameter learning may be improved by incorporating additional information provided by experts. A popular approach relies on the Maximum a Posteriori (MAP) estimation using Dirichlet priors, which express experts' belief (e.g., Heckerman et al., 1995) about $\theta$ in the absence of data. Formally, the Dirichlet distribution for CPT column $\theta_{\mathrm{ij}}$ is expressed as follows:

$p\left(\theta_{i j}\right)=\frac{1}{z_{i j}} \prod_{k=1}^{r_{i}} \theta_{i j k}\left(\alpha_{i j k}+1\right)-1$

with $\sum_{k} \theta_{i j k}=1, \theta_{i j k} \geq 0, Z_{i j}$ is a normalisation term $\int_{-\infty}^{+\infty} \prod_{k=1}^{r_{i}} \theta_{i j k}\left(\alpha_{i j k}+1\right)-1 d \theta_{i j k}=1$, and $\alpha_{i j k}$ is the parameter of the Dirichlet distribution, which can be intuitively interpreted as "how many times the expert believes he/she will observe $X_{\mathrm{i}}=\mathrm{k}$ in a sample of $\alpha_{\mathrm{ij}}$ instances drawn independently at random from the distribution $\theta_{\mathrm{ij}}$ "' (Zhou et al., 2014). On this basis, MAP relies on the following equation: the data and from the experts' prior guess. In their computer experiments using twelve publicly 
available BBNs (available at http://www.bnlearn.com/bnrepository/), Zhou et al. (2016a) showed that MAP achieves better performances than conventional MLE, which suffers from the absence of data in several state configurations in situations of limited sample size (typically $50)$.

Expert-based information can take several forms, and the one that corresponds to qualitative constraints have given rise to several developments. Instead of directly providing the exact value of the entries of binary BBN (denoted $\mathrm{P}_{1-2}$ ), the expert may feel more conformable in providing an ordering like " $\mathrm{P}_{1}>\mathrm{P}_{2}$ ", " $\mathrm{P}_{1} \approx \mathrm{P}_{2}$ ", " $\mathrm{P}_{1}>0.80$ ", etc. Zhou et al. (2016a) showed that incorporating such expert knowledge about the monotonic influences between nodes (translated into probability constraints) further outperformed MAP and MLE and was also robust to errors in labelling the monotonic influences.

Different methods have been developped to incorporate qualitative constraints, namely:

- Convex Optimization (Niculescu et al., 2006; Zhou et al., 2016a; de Campos and Ji, 2008; Liao and Ji, 2009; Altendorf et al., 2005) is an extension of the MLE by incorporating constraints via penalty functions or by restricting parameter spaces;

- Constrained MAP approach has also been proposed by Yang et al. (2019) to learn BN parameters by incorporating convex constraints;

- Isotonic Regression (Feelders and van der Gaag, 2005; 2006) builds on qualitative information about the influences between the variables of a BBN. The most recent algorithm by Masegosa et al. (2016) also enables the analysist to learn the CPT parameters from incomplete data;

- Qualitative MAP (originally proposed by Chang and Wang (2010) and further improved by Guo et al. (2017)) constructs Dirichlet priors from Monte-Carlo random samples of the constrained parameter space, which are used by the MAP algorithm;

- Multinomial Parameter Learning with Constraints (Zhou et al., 2014; Hospedales et al., 2015) rely on auxiliary BBNs, which are hybrid BBNs, to infer the posterior distribution of BBN parameters.

\subsection{Discussion}

Following a pure statistical data-driven approach for populating the BBN conditional model requires a large amount of statistically significant data to cover all BBN relationships. To compensate the lack of data, a possible option is to complement the analysis with expert-based information. Sect. 2.4 shows that a broad range of different tools/methods are available to 
incorporate expert-based information either in the form of qualitative influences or constraints, namely constraints that should be almost linear and convex (i.e. concave constraints like $\mathrm{P}_{1} \neq 0.5$ cannot be accounted for). The improvement of the learning accuracy of the parameters in BBNs from a small data set has been shown using each of the described methods compared to conventional methods; for instance Guo et al. (2017) compared MLE, constrained MLE, maximum entropy and constrained maximum entropy estimator, MAP and their qualitatively MAP estimator. Yang et al. (2019) showed the higher performance of their constrained MAP estimator compared to conventional parameter learning algorithms, MLE and MAP, and to constrained maximum likelihood algorithm. Yet, to the author's best knowledge, no extensive benchmark exercise covering all the afore-mentioned estimators (as well as their pros and cons) is available yet; practical recommendations on how to implement them and their limitations is currently lacking in the literature.

Among the possible limitations, the problem of under-fitting related to the use of prior distributions (that are common ingredients of most of the methods of Sect. 2.4) is seldom tackled. As described by Gao et al. (2019), imposing certain a priori knowledge on the CPT parameters might decrease the likelihood of the parameters, hence a reduction of the fitness between parameters and data. Azzimonti et al. (2019) proposed a hierarchical procedure to improve the widely-used approach based on Dirichlet priors. Gao et al. (2019) proposed a Minimax Fitness algorithm combined with an improved constrained maximum entropy method to overcome this problem. They also concluded that there is a need for further investigation to develop learning methods that does not require specification of prior strength.

\section{Learning from experts}

In many situation, the primary source of information for learning the CPTs is not based on data, but on inputs from expert domain. For instance, for rare-event situations like reliability analysis, inputs from expert domain stem from questionnaires, interviews and panel discussions. Sect. 3 focuses on the process of deriving information from experts that is named "elicitation". The issues and methods related to this task were analysed by review articles in different domains of application, namely shipping accidents by Zhang and Thai (2016), human reliability by Mkrtchyan et al. (2015) and more broadly regarding dependence in probabilistic modelling by Werner et al. (2017). The objective is to focus the elicitation on specific pieces of information to efficiently populate the CPTs by ensuring quality and consistency of the elicited result and minimizing the workload on the experts owing to the large number of CPT entries. Elicitation for CPT generally relies on three (possibly combined) main approaches through: (1) the 
assessment of probabilities directly from an (or a panel of) expert (Sect. 3.1); (2) assumptions

265 on the causal structure either by simplifying the network structure or by simplifying the causal dependence (Sect. 3.2); (3) filling-up methods (Sect. 3.3).

267

268

269

270

271

272

273

274

275

276

(A)

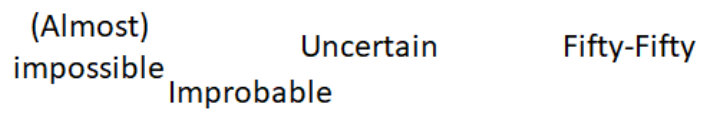

(B)

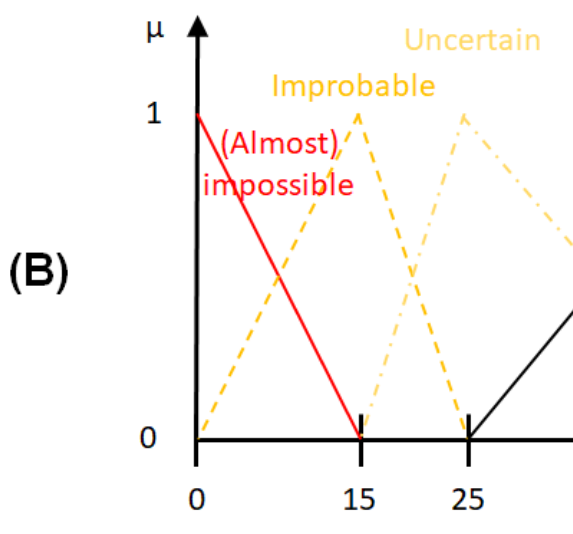

278 Figure 2. (A) Example of probability scale used to assist expert elicitation of CPTs (adapted

279 from Knochenhauer et al. (2013)); (B) Translation of the probabilities qualified in (A) into 280 Fuzzy sets ( $\mu$ is the degree of membership).

[Figure 2 about here]

283 As an alternative, experts are preferably asked to give qualitative statements (like categorical or relative measure). To support this indirect approach, tools from the domain of multicriteria 
285

286

287

288

289

290

291

292

293

294

295

296

297

298

299

300

301

302

303

304

305

306

307

308

309

310

311

312

313

314

315

316

decision-making have been proposed. For instance, Chin et al. (2009) adapted the Analytical Hierarchy Process method for the task of probability elicitation and semi-automatic generation of the parameters of CPTs. The basic idea is to elicit paired comparisons about the relative likelihood of the possible events using predefined scores (equally possible, etc.) instead of directly asking the probability values. Yet, this procedure is at the expense of an increase in the number of comparisons as the number of conditional probabilities increases.

An alternative option proposes to directly process natural linguistic terms by mathematically modelling them using for instance a Fuzzy set (Zadeh, 1975). Let us consider the concept of membership function, which defines how each element $x$ of the input space $X$ (also named "universe of discourse") is mapped to a degree of membership (denoted $\mu$ ). Under the classical theory of Boolean logic, the membership function of a set $A$ is simply defined as a binary function that takes the value $\mu(x)=1$ if the element belongs to $A$ and the value $\mu(x)=0$, otherwise. The Fuzzy set theory of Zadeh (1965) introduces the concept of a set without a crisp (i.e. clearly defined) boundary. Such a set can contain elements with only a gradual (partial) degree of membership ( $\mu$ is scaled between 0 and 1 ). The translation of the probability scale of Fig. 2(A) into Fussy sets is provided in Fig. 2(B). Some successful applications cover fault detection (D’Angelo et al., 2014), performance analysis of devices (Penz et al., 2012), safety risk analysis (Zhang et al., 2015), human reliability analysis (2012; Li et al., 2012), and offshore risk (Ren et al., 2009). Two viewpoints exist in the literature on Fuzzy BBNs. Fuzziness can be incorporated in the variables (nodes) or on the probabilities. For instance, Ren et al. (2009) carried out studies using fuzzy probability calculations in BBNs (as illustrated in Fig. 2(B)). Conversely, Tang and Liu (2007) used fuzzy events (i.e. Fuzzy node states) in BBNs for a machinery fault diagnosis problem. İçen and Ersel (2019) incorporated both aspects with application in medicine.

\subsection{Making assumptions on the causal structure}

To reduce the elicitation burden, the number of CPT entries to be elicited should be kept "reasonable". This can be performed by making assumptions regarding the causal structure. One option is by simplifying the structure through the introduction of "divorcing" nodes (Henderson et al., 2009). This involves aggregating a few of the nodes by adding a new node that summarizes them provided that the aggregations are logical and no interactions are lost in the procedure. Although this process adds nodes to the network, it reduces the combined size of CPTs in the network (Cain, 2001). Yet, divorcing might dilute the sensitivity of the final 
node(s) to the input nodes and might increase the uncertainty propagated through the network

318 as underlined by Cain (2001).

319 A popular alternative aims at making some simplifications regarding the causal dependence based on the logical Noisy-OR gate (Pearl, 1988). In their typical implementation, Noisy-OR gates focus on binary BBN nodes and assume that the influence of the considered factor is independent from the presence of the other factors. This means that the probability of the outcome is the product of the probabilities of the outcome in presence of one factor at a time, with all other factors being absent. Formally, let us consider a binary variable $Y$ with two states $\{$ False, True $\}$ and $\mathrm{n}$ binary parent variables $X_{\mathrm{i}=1, \ldots, \mathrm{n}}$.

(A)

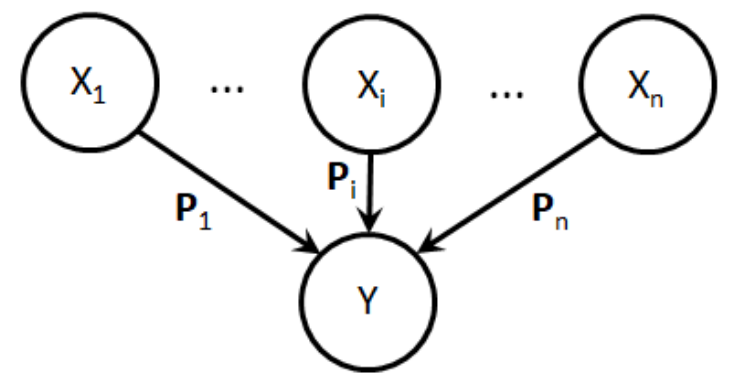

(B)

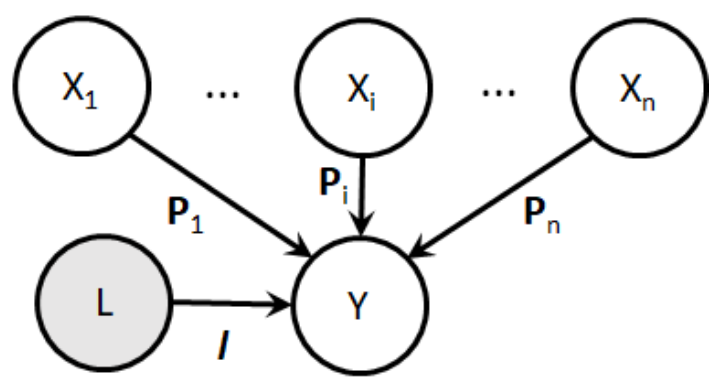

326

327

328

329

330

331

332

333

334

335

336

337

338

339

340

341

Figure 3. (A) Schematic representation of the Noisy-OR gate with $P_{\mathrm{i}=1, . ., \mathrm{n}}$ the link probabilities; (B) Schematic representation of the Noisy-OR gate.

\section{[Figure 3 about here]}

The main principle of the Noisy-OR model is to define probabilities $P_{\mathrm{i}}$ (termed as link probability, Fig. 3(A)), which are defined as the probability that $Y$ is False given that $X_{i}$ is False and $X_{j}$ is True for $i \neq j$. A Noisy-OR model is thus a disjunction "noisy" version of $X_{\mathrm{i}}$ (Pearl, 1988). This means that the distribution of $Y$ conditional on $X_{1} ; X_{2} ; \ldots ; X_{\mathrm{n}}$ is $P(Y=$ $\left.F \mid X_{1} ; \ldots ; X_{n}\right)=1-\prod_{i: X_{i} \in X_{T}}\left(1-P_{i}\right)$ where $X_{\mathrm{T}}$ is the set of parent nodes whose states are True. The Noisy-OR model enables the analysist to specify fewer CPT parameters; the number of independent parameters being here reduced from $2^{\mathrm{n}}$ to $2 \mathrm{n}$. The extension of Noisy-OR gate to multi-valued variables is the Noisy-MAX gate model (Diez, 1993; Henrion, 1989). If the parent node $X_{\mathrm{i}}$ has $n_{X_{i}}$ states, then the total number of parameters that have to be elicited using leaky Noisy-MAX gate is $N=\sum_{i=1}^{n}\left(n_{X_{i}}-1\right)\left(n_{Y}-1\right)+1$ to be compared to the total number without Noisy-MAX gate, namely $N=\left(n_{Y}-1\right) \cdot \prod_{i=1}^{n} n_{X_{i}}$. 
342 Different empirical studies have been conducted to investigate the performance of the leaky 343 Noisy-OR approach. Several authors (Oniśko et al., 2001; Anand and Downs, 2008; Bolt et al., 344 2010; among others) showed how this approach helped reducing the burden of elicitation in 345 practical real-life applications without impacting too much the performance of the network. 346 Besides, Zagorecki and Druzdzel (2012) explored to which extend the pattern of causal 347 interaction induced by Noisy-OR(MAX) gates are common in real cases. Using three existing 348 BBNs, they showed that the Noisy-MAX gate provides a good fit for as many as 50\% of CPTs 349 in two of these networks.

350 The Noisy-OR structure is based however on a strong assumption, i.e. that the node of interest 351 is in the state False (considering the above illustrative case) with a probability equal to 1 if all 352 its parent variables are in the state False. Yet, in many cases, it is often difficult to capture all the causes of the node of interest (e.g. for reliability purpose, it means to define all the failure modes of a component). To deal with this problem, Henrion (1989) proposed an extension called "leaky Noisy-OR" gate that includes a background probability that represents the influence of non-modelled causes as schematically depicted in Fig. 3(B). Zagorecki and Druzdzel (2004) proposed to elicit leaky and non-leaky Noisy-OR parameters as alternatives to conditional probabilities using statements like "What is the probability that $Y$ is present when $X_{1}$ is present and all other causes of $Y$ (including those not modelled explicitly) are absent?". They showed that the leaky Noisy-OR parameter was assessed as the most accurate (in terms of Euclidean distance to empirical distribution).

The leaky Noisy-OR method was further extended by relaxing the necessity to define a crisp precise leaky probability value, i.e. by introducing uncertainty on this parameter. This type of uncertainty has been addressed within different uncertainty treatment settings (which are introduced in more details in Sect. 4). Antonucci (2011) developed an imprecise leaky NoisyOR gate model with uncertainty on the link probabilities modelled by intervals within the formalism of credal networks (see Sect. 4.2). Alternatively, Fallet-Fidry et al. (2012) (further extended by Zhou et al. (2016b)) proposed an imprecise extensions of the Noisy-OR within the formalism of evidential networks (see Sect. 4.3). Finally, Dubois et al. (2017) developed a version of noisy logical gates within the theory of possibility (Dubois and Prade, 1988) using possibilistic causal networks (as presented by Benferhat et al. (2002)) with illustration on an example taken from human geography. 


\subsection{Filling-up methods}

374 Alternative methods to Noisy-OR(MAX) gate are based on filling-up techniques. These 375 methods are typically based on extracting information on the factor effects from known 376 relationships (named anchor conditional probability distributions, denoted CPD) and 377 extrapolating to the whole CPTs. Considering two BBNs (of respectively 3 and 4 nodes) for a human reliability problem, Mkrtchyan et al. (2015) tested five popular methods for CPT derivation considering nodes with multiple states, namely:

- Method 1: the functional interpolation method (Podofillini et al., 2014) approximate CPDs elicited at the anchor positions by functions described by parameters (e.g., Normal functions); the parameters of the missing CPDs are then obtained by interpolating those corresponding to the anchor ones;

- Method 2: the Elicitation BBN method (Wisse et al., 2008) is based on piecewise linear functions interpolating among the elicited CPDs, and on state influencing factors and importance weights;

- Method 3: The Cain calculator (Cain, 2001) uses interpolation factors derived from CPDs to populate the missing relationships in CPTs;

- Method 4: The method presented by Røed et al. (2009) is also based on functional relationships between influencing factors and outcome nodes; the parameters of the function (exponential) are then determined based on the elicitation of selected CPDs;

- Method 5: the ranked node method by Fenton et al. (2007) (further improved by Laitila and Virtanen, 2016) is not based on interpolation of known CPDs. In this approach, all the nodes are defined on the interval [0-1]. For instance, let us consider a node with 5 states, namely "very low", “low", "average”, "high”, and "very high"; each of the state is assigned to an interval width of 0.2 ; for instance, the value "low" is assigned to the interval [0.2-0.4]. To generate CPTs, the experts are asked to provide the weight parameters and to choose one algorithm (the mean average, the Minimum, the Maximum and the MixMinMax). Using this method, if there are $m$ ranked nodes and each node has $n$ states, the expert will only need $m+1$ parameter values, while it requires $\mathrm{n} \times \mathrm{m}+1$ values for full elicitation.

402 Mkrtchyan et al. (2015) showed that:

403 - All methods allow representing the different importance of the various influencing 404 factors; 
- The representation of the interactions (combined effects of multiple factors) is problematic for methods eliciting information on the influence of factors taken one at a time (methods 2-4);

- Functional representation of the CPTs (methods 1, 5 and 4) can be traced more easily, because they allow an explicit representation of uncertainty in the factor relationships;

- But methods 4 and 5 have difficulties in representing the different degrees of uncertainty in the relationships;

- The method allowing the largest modelling flexibility is method 1 with respect to strong factor influences (single and multi-factor) and proper uncertainty characterization, but becomes too costly for large BBNs.

\subsection{Discussion}

The conclusions drawn by Mkrtchyan et al. (2015) serve as valuable recommendations regarding the use and applicability of the five most popular filling-up methods for reducing the expert burden of CPT elicitation. Despite the practical usefulness of this comparative exercise, it should be noted that they primarily focused, by construction, on the modelling aspects important for their application domain (here human reliability analysis), namely the representation of strong factor influences and interactions, and the characterization of different degrees of uncertainty in the relationships. Broader exercises are needed to cover a larger spectrum of methods (i.e. filling-up methods should be completed by Noisy- OR/MAX models, direct elicitation among others), of contexts (different network sizes, binary versus multivalued nodes, etc.), as well as of domains of application.

Despite the clear advantages of these methods for BBN engineering, they cannot be applied uncritically, because the probability values can only be considered approximations of the true probabilities and whatever the considered methods, they are all based on simplifications that may hamper the BBN performance. Initiatives like the one by Woudenberg and van der Gaag (2015) for the Noisy-OR model should be intensified. They identified the conditions under which ill-considered use of this method can result in large impact on output probabilities; in particular, when the yet-unobserved cause variables in the mechanism have relatively skewed probability distributions and/or the obtained parameter probabilities have small values. For this purpose, sensitivity methods as described in Sect. 5 can play an important role. Fenton et al. (2019) also dealt with the limitations of leaky Noisy-OR model for backward inference. When the binary node of interest $\mathrm{Y}$ of the example in Fig. 3 is observed to be in the state False, the normal "explaining away" behaviour fails, which means that after observing the state of any 
438

439

440

441

442

443

444

445

446

447

448

449

450

parent the remaining parents become independent, and the results may not result in what BBN practitioners expected. Fenton et al. (2019) described a simple extension of the model that requires the elicitation of only one extra parameter that can solve this problem for a large spectrum of cases in practice.

\section{Propagating the uncertainties}

Whatever the methods used to populate the CPTs, residual uncertainties may still prevail. This residual uncertainty should be reflected in BBN-based results. This means that the uncertainty on CPT entries should be propagated in order to evaluate their consequences on the BBN results. The propagation can either rely on probabilities (Sect. 4.1), or alternative mathematical representation tools like intervals (Sect. 4.2) or a generalization of a probability distribution (Sect. 4.3), i.e. within the theory of belief functions as introduced by Shafer (1976) and Dempster (1967). Fig. 4 summarizes the main principles of the different approaches by using a simple OR-gate model.

(A)
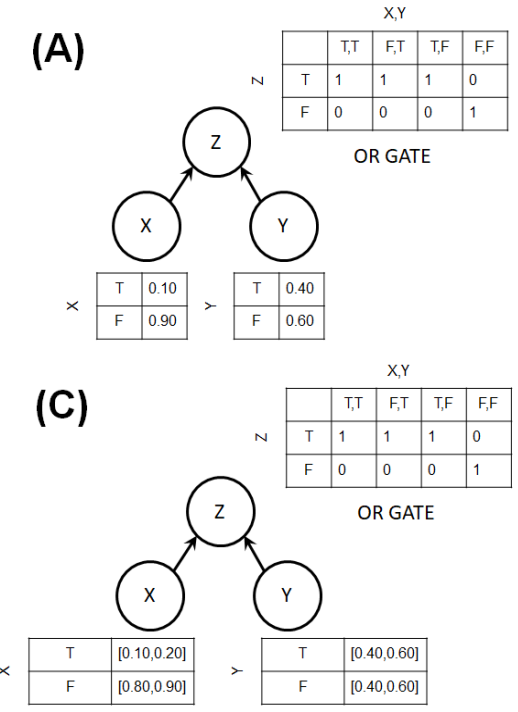

(B)

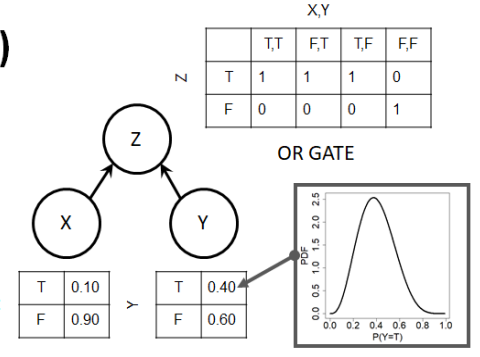

(D)

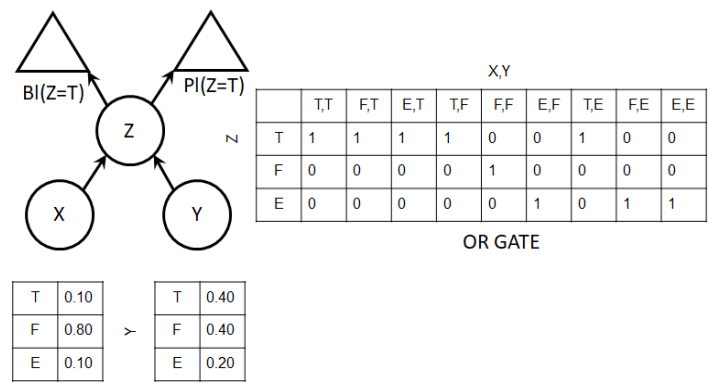

Figure 4. (A) Example of an OR gate model translated into a BBN with two binary parent nodes $X$ and $Y$ (with states corresponding to $\mathrm{T}=$ True or $\mathrm{F}=\mathrm{False}$ ). The truth table related to the $\mathrm{OR}$ gate corresponds to the table next to the child node $Z$. Illustration of a probability-based approach where uncertainties on CPT entries are represented by: (B) Beta probability distributions (with an example here for node $Y$ ); (C) Interval-valued probabilities (credal network approach); (D) Mass probability tables; here the truth table includes the epistemic state $\mathrm{E}=\{\mathrm{T}, \mathrm{F}\}$; Two nodes were added to the network to calculate the belief and plausibility functions (see Sect. 4.3 for more details). 


\subsection{Methods using probabilities}

462 The problem of uncertainty propagation for $\mathrm{BBN}$ has originally been addressed using 463 probabilities. This approach assumes that the uncertainty on CPT entries follows a Beta 464 probability distribution (or for more generic cases, a Dirichlet probability distribution), as 465 schematically depicted in Fig. 4(B). Kleiter (1996) originally described a Monte-Carlo-based random simulation procedure to carry out the approximation of the spread of the probability

467 468 469 distribution for the considered query. The method requires, however, a large number of random samples to accurately characterize the true variance. Van Allen et al. (2008) proposed an improved method by avoiding Monte Carlo sampling through the combination of bucket elimination (Dechter, 1998) with the "delta rule" that linearizes the relationship between the query probabilities and the corresponding Dirichlet conditional probabilities connecting the query variable to its parents and children. They further proved that the Beta approximation (for binary BBNs) is asymptotically valid. The conditions of the exact Beta distribution has extensively been investigated by Hooper (2008). This problem has further been formalized within the setting of subjective logic (Jøsang, 2001; 2016) as proposed by Kaplan and Ivasnoska (2018), who developed an efficient belief propagation for inference in a binary Bayesian network with a singly-connected graph. To introduce any type of probability distribution on CPTs, Fenton (2018) proposed to extend the BBN with continuous nodes corresponding to the uncertain prior probability distributions, but at the expense of a potentially large increase of computational time cost when the number of nodes and of CPTs increases.

\subsection{Methods using interval-valued probabilities}

Instead of specifying a crisp single value of each CPT entry, the formal setting of credal network, denoted CN (Cozman, 2000; 2005), integrates BBNs with credal sets, i.e. set of probability measures. A CN can be viewed as the representation of a set of BBNs, which share the same graphical structure but are associated to different conditional probability parameters; the interest being to provide a richer representation of uncertainty. In Fig. 4(C), the uncertainty in the CPTs are presented by intervals.

Formally, given a variable $X$, we denote by $\Omega$, the possibility space of $X, x$ a generic element of $\Omega, P(X)$ the probability mass function for $X$ and $P(\mathrm{x})$ the probability of $x$. The credal set over $X$ is $\mathrm{K}(X)$, which corresponds to a closed convex set of probability mass functions over $X$. For any $x \in \Omega$, the lower probability for $x$ according to the credal set $\mathrm{K}(X)$ is $\underline{P}(x)=$ 
492

493

494

495

496

497

498

499

500

501

502

503

504

505

506

507

508

509

510

511

512

513

514

515

516

517

518

519

520

$\min _{P(X) \epsilon K(X)} P(x)$. Similar expression can be given for the upper probability. Within Walley's theory of imprecise probabilities (Walley, 1991), credal sets can then be represented as polytopes, where each inner point has a valid probability mass, and can be obtained by computing the convex hull of a finite number of probabilities, called vertices (Cozman, 2000). A credal set for a random variable $X_{\mathrm{i}}$ is labelled $K\left(X_{i}\right)$, while the set comprising its extreme points is denoted by ext $\left[K\left(X_{i}\right)\right]$.

A credal network CN (Cozman, 2000) over a set of random variables is thus a DAG where dependencies among variables are defined by a set of conditional credal sets as $K\left(X_{i} \mid p a\left(X_{i}\right)\right)$. By analogy with BBN, it is possible to define a joint credal set as follows:

$K(X)=C H\left(P(X): P(x)=\prod_{i=1}^{n} P\left(x_{i} \mid p a\left(X_{i}\right)\right)\right.$

where $P\left(X_{i} \mid p a\left(X_{i}\right) \in \operatorname{ext}\left[K\left(X_{i} \mid p a\left(X_{i}\right)\right], \mathrm{CH}\right.\right.$ is the convex hull operator, applied to the probabilities computed for the combination of all the vertices of all the conditional credal sets.

In this setting, the task of inference aims at computing the probability bounds of the largest extension that satisfies the Markov condition (i.e., independence of each node of its nondescendant non-parents given its parents) under the assumption of strong independence (Cozman, 2000). This results in the convex hull of the set containing all joint distributions that factorize the overall joint probability of the network, where the conditional distributions $\mathrm{P}\left(X_{\mathrm{i}} \mid \mathrm{pa}\left(X_{\mathrm{i}}\right)=\pi_{\mathrm{k}}\right)$ are selected from the local sets $K\left(X_{\mathrm{i}} \mid \mathrm{pa}\left(X_{\mathrm{i}}\right)=\pi_{\mathrm{k}}\right)$. This task is a NP-hard (de Campos and Cozman, 2005), for which a number of exact and approximate algorithms have been proposed (Antonucci et al., 2015; Mauá et al. 2012; Ide and Cozman, 2008; Cano et al., 2007), but only exact inference algorithms are suitable to polytree-shape binary networks.

Though CN allows quantifying and integrating the uncertainty on CPTs on the BBN inference results, this increase in expressiveness comes at the expense of higher computational costs. Some real case applications of CN exist in different domains (Table 1), but the number of them remain limited (with comparison to $\mathrm{BBN}$ ), despite the availability of some open-source solutions like OpenCossan (Tolo et al., 2018), the linear programming algorithm ${ }^{1}$ of Antonucci et al. (2015), the GL2U-II algorithm² of Antonucci et al. (2010).

\begin{tabular}{|l|l|l|l|}
\hline & Domain of application & Method & Reference \\
\hline
\end{tabular}

\footnotetext{
${ }^{1}$ http://ipg.idsia.ch/software.php?id=135

2 http://ipg.idsia.ch/software.php?id=142
} 


\begin{tabular}{|c|c|c|c|}
\hline 1 & Hazard assessment of debris flows & $\mathrm{CN}$ & Antonucci et al. (2007) \\
\hline 2 & Military identification & $\mathrm{CN}$ & Antonucci et al. (2009) \\
\hline 3 & $\begin{array}{l}\text { Reliability analysis of a fire-detector } \\
\text { system }\end{array}$ & EN & $\begin{array}{l}\text { Simon et al. (2008; 2009; } \\
\text { 2017) }\end{array}$ \\
\hline 4 & Threat assessment & EN & Benavoli et al. (2009) \\
\hline 5 & Convoy detection & EN & Pollard et al. (2010) \\
\hline 6 & $\begin{array}{l}\text { Reliability analysis of oil filter plug } \\
\text { linked to aero engines }\end{array}$ & EN & Yang et al. (2012) \\
\hline 7 & Railway dysfunction & EN & Aguirre et al. (2013) \\
\hline 8 & Food processing & Dynamic CN & Baudrit et al. (2016) \\
\hline 9 & Cyber attack analysis & EN & Friedberg et al. (2017) \\
\hline 10 & $\begin{array}{l}\text { Vulnerability analysis of Nuclear } \\
\text { Power Plant subject to external hazards }\end{array}$ & $\mathrm{CN}$ & Tolo et al. (2017) \\
\hline 11 & $\begin{array}{l}\text { Reliability analysis of a safety } \\
\text { instrumentation system } \\
\text { pressurized vessel }\end{array}$ & EN & Zhang et al. (2017) \\
\hline 12 & Medical prognostic and diagnostic & $\begin{array}{l}\text { EN and } \\
\text { Fuzzy sets }\end{array}$ & $\begin{array}{l}\text { Janghorbani and Moradi } \\
\text { (2017) }\end{array}$ \\
\hline 13 & Fault diagnosis for railway & BBN vs EN & Verbert et al. (2017) \\
\hline 14 & Maritime accidents & $\mathrm{CN}$ & Zhang and Thai (2018) \\
\hline 15 & $\begin{array}{l}\text { Terrorist attack analysis on a chemical } \\
\text { storage plant }\end{array}$ & $\mathrm{EN}$ vs $\mathrm{CN}$ & Misuri et al. (2018) \\
\hline 16 & Landslides & $\mathrm{CN}$ & He et al. (2018) \\
\hline 17 & $\begin{array}{l}\text { Risk assessment of an oscillating water } \\
\text { column }\end{array}$ & $\mathrm{CN}$ & Estrada-Lugo et al. (2018) \\
\hline 18 & $\begin{array}{l}\text { Reliability analysis of a feeding control } \\
\text { system }\end{array}$ & EN & Mi et al. (2018) \\
\hline 19 & $\begin{array}{l}\text { Human reliability for Nuclear Power } \\
\text { Plant safety analysis }\end{array}$ & EN & Deng and Jiang (2018) \\
\hline 20 & Safety assessment of a truss & EN & Khakzad (2019) \\
\hline
\end{tabular}

Table 1. Case studies of evidential networks (EN) and Credal Network (CN)

\subsection{Methods based on Dempster-Shafer Theory}

525 An alternative setting for representing imprecision is the theory of belief functions, also called 526 Dempster-Shafer Theory, denoted DST (Shafer, 1976, Dempster, 1967). Let $X$ be a variable 527 taking values in the frame of discernment $\Theta$ composed of $q$ mutually and exhaustive possible 528 state of $X$. For instance, for a binary node, the frame of discernment is $\Theta=\{$ True, False . 
529 Formally, the theory introduces the concept of basic belief assignment (BBA) based on the 530 belief mass function $m: 2^{\Theta} \rightarrow[0,1]$ and satisfies $\sum_{A \subseteq \Theta} m(A)=1$, and $m(\varnothing)=0$ (which 531 assumes that at least one element of $\Theta$ is true). Every $A \in 2^{\Theta}$ such that $\mathrm{m}(A)>0$ is called a focal 532 element.

533 In classical probabilities, a probability value can be assigned to the state True or False only. By 534 defining the belief mass function based on the powerset of the frame of discernment $2^{\Theta}$ (which 535 corresponds in the binary example to $\{\varnothing$,True, False, $\{$ True,False $\}\}$ ) enables the analysist to 536 allocate a quantity supporting an additional state termed as epistemic state $E=\{$ True, False $\}$. 537 Due to uncertainty, the analysist may not always be able to determine the amount of masses to 538 attribute to each state, and the variable $X$ may then be in both states, True or False. This means 539 that the method allows characterizing uncertainty about the state of a given node.

540 From a mass function $m$, two measures can be defined (instead of one for the probabilistic case) 541 called the belief $(B l)$ and plausibility $(P l)$ measures. The latter are respectively defined, for any event $A$ as follows:

$544 \quad B l(A)=\sum_{E \subseteq A} m(E)$, and $\operatorname{Pl}(A)=\sum_{E \cap A \neq \varnothing} m(E)$

545 where $B l$ measures how much event $A$ is implied by the information (it sums masses that must 546 be redistributed over elements of $A$ ), $P l$ measures how much event $A$ is consistent with the 547 information (it sums masses that could be redistributed over elements of $A$ ). These two 548 measures can be associated to a (closed convex) set of bound probabilities $\{P \mid \forall A \subseteq \Theta,(A)$ $549 \leq(A) \leq P l(A)\}$. It is thus possible to associate an interval-valued probability to the event $A$, with 550 minimum and maximum probabilities provided by $\mathrm{Bel}$ and $\mathrm{Pl}$, respectively. This makes the 551 formal link with $\mathrm{CN}$. Conversely, it is also possible to reconstruct BBAs from $\mathrm{Pl}$ and $\mathrm{Bel}$ 552 functions using a Möbius Transformation (Smets, 2002).

553 As an illustration, let us assume consider a binary node for which the expert only knows that 554 the probability of the event $\{X=$ True $\}$ is at least 0.8 . The corresponding BBA is $m(\{$ True $\})=0.8$, 555 $m(E=\{$ True, False $\})=0.2, m(\{$ False $\})=0$. This means that $B l(\{$ True $\})=m(\{$ True $\})$, and $P l(\{$ True $\})=m(\{$ True $\})+m(E)=0.8+0.2=1.0$. This also means that $B l(\{$ True $\})=m(\{$ True $\})=0.8$, and $P l(\{$ True $\})=\mathrm{m}(\{$ True $\})+m(E)=0.8+0.2=1.0$. Then $0.8 \leq \mathrm{P}(\{$ True $\}) \leq 1.0$.

558 The evidence theory is the basis of evidential networks (EN), which is a DAG propagating 559 belief masses. One of the first formulation by Xu and Smets (1996) is based on the Dempster's 
560

561

562

563

564

565

566

567

568

569

570

571

572

573

574

575

576

577

578

579

580

581

582

583

584

585

586

587

588

589

590

591

rule for combining and reasoning with the belief masses. Yet, one major limitation is that the inference algorithms in this formulation are less effective that the one for traditional BBNs as underlined for instance by Khakzad (2019). In the domain of system reliability analysis, Simon et al. (2008) proposed an alternative by mapping logical gates (like OR or AND typically used for fault tree analysis), as EN with the hypothesis described by Guth (1991). Despite its similarity with $\mathrm{BBN}$, relations in $\mathrm{EN}$ between variables are not probabilities, but belief masses. The truth table of gates are replaced by conditional mass tables for AND and OR gates (see an example in Fig. 4(D)). To compute belief and plausibility measures in EN, specific nodes (as proposed by Simon and Weber 2009) are introduced. Three types of nodes (as represented in Fig. 4(D)) are thus defined (Simon and Bicking, 2017), namely:

- Root nodes to which BBA are assigned, correspond to components;

- Non-root nodes correspond to logical gates that encode its resulting states \{True, False, \{True,False\} given the states of its parents;

- Evaluation nodes correspond to nodes that aim at providing estimates of the belief and plausibility measures of the system state.

In the formulation by Simon and Weber (2009), the inference computation is based on the Bayes theorem, which is extended to DST by specifying a mass of 1 on one of the focal elements of the frame of discernment for a specific evidence (hard evidence). Non-specific evidence (soft evidence) corresponds to a mass distribution on the focal elements of the frame of discernment. This means that probability updating in such EN can be based on BBN inference algorithms.

Misuri et al. (2018) compared CN and EN with illustration on a terrorist attack analysis on a chemical storage plant. They highlighted that:

- When used for uncertainty propagation, EN and CN give the same results;

- In terms of implementation, EN is simplier to use, because they can be built using existing codes for $\mathrm{BBN}$, whereas $\mathrm{CN}$ requires specific codes;

- In terms of interpretation, Misuri et al. (2018) concluded that EN is more intuitive, because experts directly assign some weight to the epistemic state (e.g. $E=\{$ True,False $\}$ for a binary node), whereas they have to specify interval-valued probabilities for $\mathrm{CN}$, which can become tricky for multivalued nodes.

Khakzad (2019) further filled the gap between $\mathrm{CN}$ and EN by proposing some heuristic rules to determine prior belief masses based on imprecise probabilities. They further modified Simon and co-authors' EN formulation to both improve the propagation and updating of the belief 
592

593

594

595

596

597

598

599

600

601

602

603

604

605

606

607

608

609

610

611

612

613

614

615

616

617

618

619

620

621

622

623

624

masses using BBNs. In order to deal with linguistic variables for the network node' states, the EN method can be combined either with Fuzzy sets (Zadeh, 1975) as applied by Janghorbani and Moradi (2017) for medical prognostic, or with a Naive Bayes classifier model as applied by Zhang et al. (2017) for safety analysis for nuclear power plant.

\subsection{Discussion}

Different settings are available to help the BBN analysist to deal with the problem of uncertainty propagation. A natural question is the justification for using approaches that are alternative to classical probabilities. A first argument often highlights the epistemic nature of the CPT uncertainties. Contrary to aleatory uncertainty (also referred to as randomness), which represents the variability of the physical environment or engineered system under study, epistemic uncertainty mainly stems from the incomplete/imprecise nature of available information (e.g. Hoffman and Hammonds, 1994). While tools from the probabilistic setting can appropriately handle aleatory uncertainties, it is the second type, which raises several problems in practice. In our situation, probability distribution cannot be inferred from data/observations, and should therefore be assumed; the procedure described in Sect. 4.1 is mainly based on the assumption that the uncertainties on the CPTs are described by a Beta (or for more generic cases, a Dirichlet probability distribution) probability distribution. Yet, this assumption may influence the final results of the BBN-based analysis (see. e.g., Ditlevsen, 1994 for an extensive discussion in reliability analysis); Relying only on probabilities masks this problem and might induce an appearance of more refined knowledge with respect to the existing uncertainty than is really present (Klir, 1989; 1994). Sect. 4.2 and 4.3 describe alternative nonprobabilistic frameworks to represent uncertainty in situations characterized by limited available pieces of information, which are mainly restricted to expert judgements. Both approaches allow improving the expressiveness with respect to uncertainty representation (as shown by the few tens of application studies using these techniques, see Table 1), in particular by enabling the $\mathrm{BBN}$ analysist to translate his/her uncertainty on the node states or his/her imprecision on the CPT parameters by avoiding the need for specifying a probability model.

Yet, extra-probabilistic approaches (whatever the considered methods, CN, EN or networks combined with linguistic variables or based on alternative uncertainty theories like possibility theory, see Dubois et al. (2017)) might come at the expense of higher level of sophistication and of complexity of the inference algorithms (and potentially higher computational costs). The danger is to add more confusion than insights as discussed by Aven and Zio (2011) with the viewpoint of decision making for risk management. The question of selecting the most 
625

626

627

628

629

630

631

632

633

634

635

636

637

638

639

640

641

642

643

644

645

646

647

648

649

650

651

652

653

654

appropriate approaches for representing and characterizing the risk and uncertainties (in particular with application on BBNs) still remains open (see e.g., an extensive discussion by Flage et al. 2014).

\section{Characterizing the uncertainties}

Methods presented in Sect. 4 allows evaluating the impacts of CPT uncertainties on the BBN results. But, this tells nothing about the respective contribution of the different CPT entries on the total uncertainty, i.e. the influence of the different uncertainties. This is the purpose of sensitivity analysis (SA), which can be used, in the construction phase of the BBN model, to study how the output of a model varies with variation of the CPT parameters. Subsequently, the results from SA can be used as a basis for parameter tuning, as well as for studying the robustness of the model output to changes in the parameters (Coupé and van der Gaag, 2002; Laskey et al., 1995).

\subsection{Description of the methods}

For discrete BBNs, a widespread SA method relies on the use of sensitivity functions (Coupé and van der Gaag, 2002; Castillo et al., 1997), which describe how the considered output probability varies as one CPT entry value is changed. An example of application in the domain of marine safety is provided by Hänninen and Kujala (2012). Formally, consider the conditional probability $\mathrm{P}(Z=k \mid e)$, where $e$ denotes the available evidence, and a CPT entry $x=\mathrm{P}(X=\mathrm{i} \mid \pi)$ where $i$ is a value of a variable $X$ and $\pi$ is a combination of values for the parents of $X$. The sensitivity function then corresponds to a quotient of two functions that are linear in $x$ of the following form:

$f(x)=\frac{c_{1} x+c_{2}}{c_{3} x+c_{4}}$

where the constants $c_{\mathrm{i}}$ are built from the values of the network's non-varied parameters. The numerator of Eq. 6 expresses the joint probability $\mathrm{P}(Z=k \mid e)$ as a function of $x$, and its denominator describes $\mathrm{P}(e)$ in terms of $x$. Using the example described in Fig. 1, we focus on the probability of having brain tumor given absence of coma but increased level of serum calcium, i.e. $P(B=T \mid C=F, I S C=T)$. Van der Gaag et al. (2013) estimated the sensitivity of this probability of interest to the probability of having coma given absence of brain tumor but increased level of serum calcium, $x=P(C=T \mid B=F, I S C=T)$. The sensitivity function 
was established as $\frac{-0.03}{x-1.03}$ (as depicted in Fig. 5(A)). This type of function shows that the 656 probability of interest steeply increases when $x$ exceeds 0.80 , i.e. above the original parametrization given by Cooper (1984).

(A)

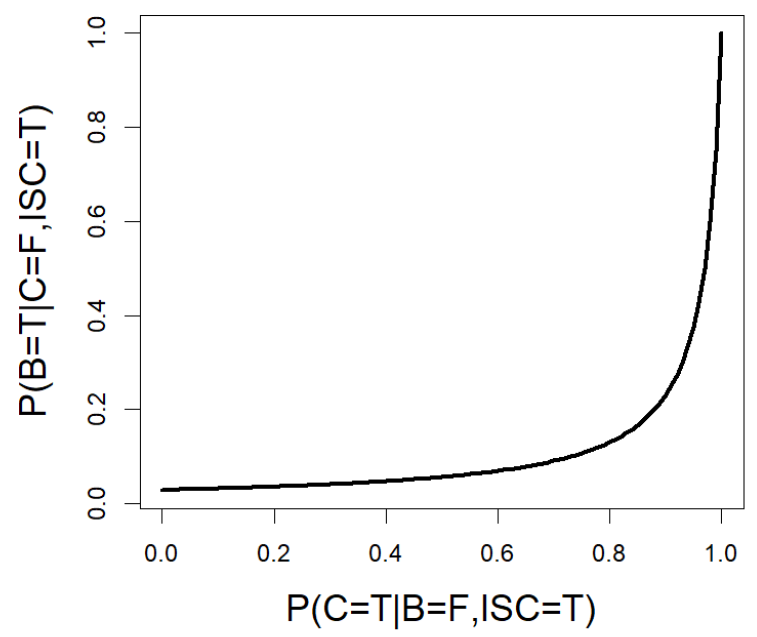

(B)

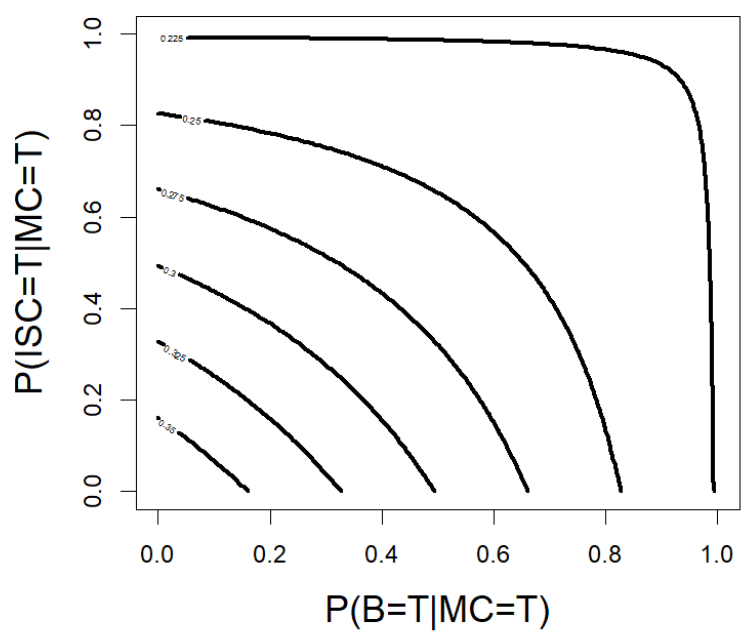

658

659

660 661

662

663

664 665 666

667

668

669

670

$f(x, y)=\frac{c_{1} x \cdot y+c_{2} x+c_{3} y+c_{4}}{c_{5} x \cdot y+c_{6} x+c_{7} y+c_{8}}$

671 where the constants $c_{\mathrm{i}}$ are estimated from the values of the network's non-varied parameters. 672 In the tumor BBN example (Fig. 1), we focus on the probability of having a cancer $\mathrm{P}(C=T)$ and 673 its sensitivity to the simultaneous variation of the conditional probabilities $x=\mathrm{P}(B=T \mid M C=T)$ 674

Figure 5. (A) One-way sensitivity function for the BBN described in Fig. 1; (B) Two-way sensitivity function for the BBN described in Fig. 1 considering the probability $\mathrm{P}(\mathrm{C}=\mathrm{T})$ as the targeted probability.

[Figure 5 about here]

Two-way sensitivity functions can be expressed in a similar form as a quotient of two bi-linear functions. Consider the sensitivity function of $f(x, y)$ that expresses $\mathrm{P}(\mathrm{Z}=k \mid e)$ as a function of the parameter probabilities $x=P\left(X=i \mid \pi_{X}\right)$ and $y=P\left(Y=j \mid \pi_{D}\right)$, where $\mathrm{i}$ and $\mathrm{j}$ are values of the variables $X$ and $Y$, and $\pi_{X}$ and $\pi_{Y}$ are combinations of values for the parents of $X$ and of $Y$. The function holds as follows:

and $y=\mathrm{P}(I S C=T \mid M C=T)$, which was established by van der Gaag et al. (2013) as $0.374+$ 
675 0.15.x.y - 0.15.x-0.15.y (Fig. 5(B)). This type of function shows that despite the large 676 variation of $x$ and $y$ (from 0 to 1 ), the probability of interest varies over a moderate range of 677 values of only $\sim 15 \%$.

678 A complementary approach for SA involves the study of the Chan-Darwiche (CD) distance 679 (Chan and Darwiche, 2002; 2005), which is a measure for bounding probabilistic belief change. 680 It is complementary in the sense that it gives insight in the effect of parameter changes on the global joint distribution, rather than on a specific (posterior) output probability of interest (as sensitivity functions do). In practices, the CD distance can be used to identify parameter changes, which lead the closer distance between the original and the varied BBN distributions (Chan and Darwiche, 2005). It should however be noted that the choice of the type of distance is rather arbitrary as outlined by Renooij (2014) and other distances like the KL-divergence (Kullback and Leibler, 1951) or the $\phi$-divergences (Ali and Silvey, 1966) could also be of interest.

Recent studies have focused on the properties of the SA methods. Renooij (2014) thoroughly investigated the different schemes for varying a probability from a (conditional) distribution, while co-varying the remaining probabilities from the same distribution; the proportional covariation scheme being the most popular one. Leonelli et al. (2017) further formalized the SA problem for discrete BBNs within the generic setting of multilinear models. They developed a unifying approach to sensitivity methods via the interpolating polynomial representation of discrete statistical models in the context of "BBNs single full CPT analyses", i.e. where one parameter from each CPT of one vertex of a BBN given each configuration of its parents is varied. This approach based on multilinear probabilistic models enabled them to address the problem of multi-way SA (with dimension $\geq 2$ ). Furthermore, they proved the optimality of proportional covariation by showing that the $\mathrm{CD}$ distance is minimized when parameters are proportionally co-varied.

\subsection{Discussion}

Since the nineties, the BBN community has seen the developments of SA methods that are specifically dedicated to their respective needs regarding the BBN use and application. Though simple and efficient to implement, the approach based on sensitivity functions (combined with CD-distance analysis) remains local, because one parameter values are varied, while the other ones are kept constant. Multi-way SA methods have been proposed, but can rapidly become intractable. Interestingly, outside the BBN community, the problem of SA is commonly addressed with alternative tools; variance-based global SA techniques being the most popular 
one (Iooss and Lemaitre, 2015). Such techniques were adapted by Li and Mahadevan (2018) to

709 bridge the gap between both communities. Their approach has the advantage to be global i.e.

710 in the sense that all CPT parameters' values are changed all together. Besides, this approach

711 can be applicable to any types of BBN (discrete, hybrid or continuous), i.e. it is model-free.

\section{Concluding remarks}

\section{$713 \quad 6.1$ Summary}

714 The current survey has investigated how to deal with uncertainties related to the specification 715 of CPTs for discrete BBNs. Three questions were addressed, namely: (1) how to constrain the 716 uncertainties related to CPT derivation; (2) how to integrate these uncertainties in the BBN717 based analysis; (3) how to test the robustness of the BBN-based results to these uncertainties.

718 Table 2 provides a summary of the main methods/approaches (together with their advantages 719 and limits) to answer these questions.

\begin{tabular}{|c|c|c|c|c|}
\hline Question & Approach & Advantages & Limits & Section \\
\hline 1 & $\begin{array}{lrr}\text { Learning } & \text { CPT } & \text { by } \\
\text { combining } & \text { data } & \text { and } \\
\text { expert } & & \text { prior } \\
\text { knowledge via } & \text { MAP } \\
\text { estimation } & & \end{array}$ & $\begin{array}{l}\text { - It improves the } \\
\text { MLE-based fitting } \\
\text { when the number of } \\
\text { data is limited. }\end{array}$ & $\begin{array}{l}\text { - The representation of } \\
\text { expert belief is } \\
\text { restricted to the use } \\
\text { of Dirichlet priors; } \\
\text { There is a possible } \\
\text { problem of "under- } \\
\text { fitting" in sparse } \\
\text { situations. }\end{array}$ & $\begin{array}{l}\text { Sect. } 2.4- \\
2.5\end{array}$ \\
\hline 1 & $\begin{array}{l}\text { Learning CPT by } \\
\text { combining data and } \\
\text { qualitative constraints }\end{array}$ & $\begin{array}{l}\text { - The accuracy of the } \\
\text { MLE/MAP-based } \\
\text { fitting is largely } \\
\text { improved when data } \\
\text { are scarce; } \\
\text { The experts may feel } \\
\text { more conformable in } \\
\text { providing ordering } \\
\text { than precise CPT } \\
\text { values. }\end{array}$ & $\begin{array}{l}\text { - Many new estimators } \\
\text { are available, but } \\
\text { many lack practical } \\
\text { recommendations; } \\
\text { - There is a possible } \\
\text { problem of "under- } \\
\text { fitting" depending on } \\
\text { the chosen priors. }\end{array}$ & $\begin{array}{l}\text { Sect. 2.4- } \\
2.5\end{array}$ \\
\hline 1 & $\begin{array}{ll}\text { Direct } & \text { elicitation } \\
\text { using } & \text { qualitative } \\
\text { statements } & \end{array}$ & $\begin{array}{l}\text { - The experts may feel } \\
\text { more conformable in } \\
\text { providing qualitative } \\
\text { statements than } \\
\text { quantitative } \\
\text { estimates. }\end{array}$ & $\begin{array}{l}\text { Mathematical } \\
\text { modelling of } \\
\text { linguistic terms may } \\
\text { lead to information } \\
\text { loss or increased } \\
\text { computation burden. }\end{array}$ & Sect. 3.1 \\
\hline 1 & $\begin{array}{l}\text { Use of "divorcing" } \\
\text { nodes }\end{array}$ & $\begin{array}{l}\text { The number of nodes } \\
\text { is decreased through } \\
\text { aggregation of nodes. }\end{array}$ & $\begin{array}{l}\text { - Care should be paid } \\
\text { to avoid the loss of } \\
\text { interactions in the } \\
\text { procedure; } \\
\text { - It may dilute the } \\
\text { sensitivity of the } \\
\text { final node(s) to the } \\
\text { input nodes; }\end{array}$ & Sect. 3.2 \\
\hline
\end{tabular}




\begin{tabular}{|c|c|c|c|c|}
\hline & & & $\begin{array}{l}\text { - It might increase the } \\
\text { uncertainty } \\
\text { propagated through } \\
\text { the BBN. }\end{array}$ & \\
\hline 1 & $\begin{array}{l}\text { Simplification of the } \\
\text { causal structure using } \\
\text { logical gates (e.g. } \\
\text { Noisy-OR gate) }\end{array}$ & $\begin{array}{l}\text { - The number of nodes } \\
\text { to be elicited is } \\
\text { largely decreased } \\
\text { (e.g. from from } 2^{\mathrm{n}} \text { to } \\
2 \mathrm{n} \text { for a binary node } \\
\text { with n parents). }\end{array}$ & $\begin{array}{l}\text { - The assumptions on } \\
\text { the causal } \\
\text { relationships might } \\
\text { not always be valid in } \\
\text { real life applications; } \\
\text { The simplifications } \\
\text { may hamper the } \\
\text { BBN performance. }\end{array}$ & Sect. 3.2 \\
\hline 1 & $\begin{array}{l}\text { Extracting } \\
\text { information on the } \\
\text { factor effects from } \\
\text { known relationships } \\
\text { and extrapolating } \\
\text { them }\end{array}$ & 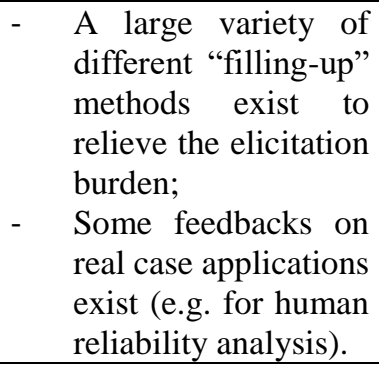 & $\begin{array}{l}\text { Simplifications are } \\
\text { introduced and the } \\
\text { derived probabilities } \\
\text { can only be } \\
\text { considered } \\
\text { approximations of } \\
\text { the true probabilities. }\end{array}$ & Sect. 3.3 \\
\hline 2 & $\begin{array}{l}\text { Uncertainty } \\
\text { propagation using } \\
\text { probabilities }\end{array}$ & $\begin{array}{l}\text { The degree of } \\
\text { confidence in the } \\
\text { BBN-based results } \\
\text { can be quantified. }\end{array}$ & $\begin{array}{l}\text { The uncertainty } \\
\text { representation is } \\
\text { restricted to the use } \\
\text { of Beta/Dirichlet } \\
\text { probability } \\
\text { distributions; } \\
\text { It can become } \\
\text { computationally } \\
\text { intensive. }\end{array}$ & Sect. 4.2 \\
\hline 2 & $\begin{array}{l}\text { Uncertainty } \\
\text { propagation using } \\
\text { intervals with credal } \\
\text { networks }(\mathrm{CN})\end{array}$ & $\begin{array}{l}\text { - It avoids selecting a } \\
\text { probability model to } \\
\text { represent the } \\
\text { uncertainty; } \\
\text { The experts may feel } \\
\text { more comfortable in } \\
\text { assigning intervals } \\
\text { than probabilities. }\end{array}$ & $\begin{array}{l}\text { - The specification of } \\
\text { interval-valued } \\
\text { probabilities can } \\
\text { become tricky for } \\
\text { multivalued nodes; } \\
\text { It needs specific } \\
\text { sophisticated } \\
\text { inference algorithms } \\
\text { and software } \\
\text { solutions (with } \\
\text { potential high } \\
\text { computational costs). }\end{array}$ & Sect. 4.3 \\
\hline 2 & $\begin{array}{l}\text { Uncertainty } \\
\text { propagation within } \\
\text { the Dempster-Shafer } \\
\text { Theory by using } \\
\text { evidential networks } \\
\text { (EN) }\end{array}$ & $\begin{array}{l}\text { - The expressiveness } \\
\text { is improved like for } \\
\text { CN; } \\
\text { EN is more intuitive } \\
\text { than CN, because } \\
\text { experts directly } \\
\text { assign some weight } \\
\text { to the epistemic state; } \\
\text { It can be } \\
\text { implemented with } \\
\text { existing } \\
\text { softwares. }\end{array}$ & $\begin{array}{l}\text { - The translation of } \\
\text { interval-valued } \\
\text { probabilities within } \\
\text { this setting can } \\
\text { become difficult for } \\
\text { multivalued nodes; } \\
\text { The inference } \\
\text { algorithms for } \\
\text { combining } \\
\text { joint/disjoint belief } \\
\text { masses are not so } \\
\text { effective as those } \\
\text { based on probability } \\
\text { theory. }\end{array}$ & Sect. 4.4 \\
\hline 3 & $\begin{array}{l}\text { Sensitivity Analysis } \\
\text { (SA) using sensitivity } \\
\text { functions }\end{array}$ & $\begin{array}{l}\text { - The theory is well- } \\
\text { established; } \\
\text { - It is simple to } \\
\text { implement; }\end{array}$ & $\begin{array}{l}\text { It focuses on the } \\
\text { influence of one (or } \\
\text { multiple) CPT } \\
\text { parameters while the }\end{array}$ & Sect. 5.1 \\
\hline
\end{tabular}




\begin{tabular}{|c|c|c|c|c|}
\hline & & $\begin{array}{llr}\text { - } & \text { The graphical } \\
\text { representation is } \\
\text { straightforward to } \\
\text { interpret. }\end{array}$ & $\begin{array}{l}\text { other ones are kept } \\
\text { constant; } \\
\text { - It requires specific } \\
\text { co-variations } \\
\text { schemes; } \\
\text { Multi-way SA can } \\
\text { rapidly become } \\
\text { intractable. }\end{array}$ & \\
\hline 3 & $\begin{array}{l}\text { Sensitivity Analysis } \\
\text { using Chan- } \\
\text { Darwiche distance }\end{array}$ & $\begin{array}{l}\text { - It complements the } \\
\text { sensitivity functions } \\
\text { by giving insight in } \\
\text { the effect of } \\
\text { parameter changes } \\
\text { on the global joint } \\
\text { distribution. }\end{array}$ & $\begin{array}{l}\text { - It presents the same } \\
\text { disadvantages than } \\
\text { the sensitivity } \\
\text { functions; } \\
\text { - The choice of the } \\
\text { distance can be rather } \\
\text { arbitrary. }\end{array}$ & Sect. 5.1 \\
\hline
\end{tabular}

Table 2. Summary of the advantages and limits of the main approaches

723 Considering the first question, we described methods for deriving CPT entries from different sources of information (observations, prior knowledge, expert-based information, etc.). Traditional estimators like MLE and MAP (or new ones) were proposed to make the best use of the data available even in scarce situations when completed by qualitative constraints like knowledge about the monotonic influences between nodes. For rare-event situations like reliability analysis, the main source of information relies on inputs from expert domain using different elicitation techniques; the main challenge being the minimization of the workload on the experts owing to the large number of CPT entries while preserving the quality and consistency of the elicited result. Elicitation for CPTs generally relies on three (possibly combined) main approaches: (1) through the assessment of probabilities directly from an expert or a panel of experts; (2) through a simplification of the causal structure using the popular Noisy-OR(MAX) model (and its improved versions like the leaky one); (3) through filling-up methods, which have in particular been thoroughly benchmarked on test cases in the domain of human reliability analysis.

737 The second question can be addressed using different approaches, either using probabilities, or imprecise probabilities either using interval-valued probabilities within the setting of credal networks or within the Dempster-Shafer theory within the setting of evidential networks. Though the latter approach enables an increase in expressiveness with respect to uncertainty representation (as shown by the few tens of application studies using these techniques, see Table 1), this might come however at the expense of higher complexity of the inference algorithms (and higher computational costs). Finally, the third question is investigated by methods specifically developed for sensitivity analysis of BBN; in particular through the use of one- or multi- way sensitivity functions. 


\subsection{Discussion and open questions}

$747 \mathrm{BBN}$ is now viewed as a suitable tool for overcoming data gaps, estimating uncertainties, and 748 visualizing complex causal relationships. Despite its clear advantages, it cannot be applied 749 uncritically, and addressing the question of uncertainties in its construction, and more 750 specifically in the CPT derivation, should become standard practices to increase the confidence in its use. The analysis of the literature (Table 2) shows that any analysist is now equipped with a handful of different tools/methods to address the question of uncertainties. This participates to the minimization of the concern of Neil et al. (2000): "In the literature much more attention is given to the algorithmic properties of BBNs than to the method of actually building them in practice".

Yet, the developments of these techniques is only one part of the problem, and effort should be intensified to bring them to an operative state. To this purpose, the implementation of these techniques within commonly-used BBN software packages (like Beuzen and Simmons 2019 for the widely-used Netica software, Norsys Software Corp., 2006 or Tolo et al. (2018) who proposed an open-source software package OpenCossan) should be strengthened. Second many methods lack practical recommendations. Therefore, more benchmark / comparative exercises are needed to cover broader situations and to serve as best practices for selecting the most appropriate tools depending the characteristics of the considered situation. For instance, the filling-up methods benchmarked by Mkrtchyan et al. (2015) should be completed by NoisyOR/MAX models, direct elicitation among others, and applied in different contexts (different network sizes, binary versus multivalued nodes, etc.), as well as domains of application. Similarly, there is a need for comparing the pros and cons of using alternative frameworks for uncertainty representation and propagation in BBNs, i.e. comparing approaches using probabilities, or interval-valued or Dempster-Shafer structures or possibility distributions or Fuzzy sets, for instance by following the initiatives conducted for probabilistic risk analysis (e.g., Pedroni et al., 2013, Loschetter et al., 2016).

The current work has focused on CPT derivation for discrete BBN development. The second key ingredient of BBNs is the DAG specification, whose learning from data has been investigated in numerous studies (e.g., Heinze-Deml et al. (2018), Scutari et al. (2018), Beretta et al. (2018), etc.). To address the whole spectrum of uncertainties in BBN building, studies both covering DAG and CPT learning would be beneficial. To integrate both sources of uncertainty, possible lines of future research may either focus on the improvement of existing algorithm like the structural expectation-maximization algorithm (Benjumeda et al., 2019) to simultaneously learn the structure and parameters of a $\mathrm{BN}$ from incomplete data, or on the 
780 combination/aggregation of multiple BBNs, each of them being based on a different set of 781 assumptions either regarding structure or CPT parametrisation (Kim and Cho, 2017; Feng et 782 al., 2014).

783 Finally, it should be underlined that BBN modelling is a rapidly advancing field (see e.g., 784 Marcot and Penman, 2019) that covers new applications and features (like the incorporation of 785 the time and space dimension, the improvements in the treatment of discrete and continuous 786 variables, its links with artificial intelligence, among others). The research on the uncertainty 787 treatment for these new developments is active (see e.g., recent advances for sensitivity analysis 788 of a wide array of graphical models by Leonelli (2019)), and the scope of the current work 789 should be broadened in the future to include them.

\section{Acknowledgements}

792 This study has been carried out within the NARSIS project, which has received funding from 793 the European Union's H2020-Euratom Programme under grant agreement $N^{\circ} 755439$. 


\section{References}

Aguirre, F., Sallak, M., Vanderhaegen, F., Berdjag, D., 2013. An evidential network approach to support uncertain multiviewpoint abductive reasoning. Information Sciences, 253, 110-125.

Ali, S.M., Silvey, S.D., 1966. A general class of coefficients of divergence of one distribution from another J. R. Stat. Soc. Ser. B, 28, 131-142.

Altendorf, E.E., Restificar, A.C., Dietterich, T.G., 2005. Learning from sparse data by exploiting monotonicity constraints. In: Proceeding of the 21st Conf. Uncertainty in Artificial Intelligence, pp. 18-26.

Anand, V., Downs, S. M., 2008. Probabilistic asthma case finding: a noisy or reformulation. In: Annual AMIA Symposium, pp. 6-10.

Antonucci, A., 2011. The Imprecise Noisy-OR Gate. In: Proceedings of the 14th International Conference on Information Fusion, 709-715, Chicago.

Antonucci, A., Brühlmann, R., Piatti, A., Zaffalon, M., 2009. Credal networks for military identification problems. International Journal of Approximate Reasoning, 50(4), 666-679.

Antonucci, A., de Campos, C.P., Huber, D., Zaffalon, M., 2015. Approximate credal network updating by linear programming with applications to decision making. International Journal of Approximate Reasoning, 58, 25-38.

Antonucci, A., Salvetti, A., Zaffalon, M., 2007. Credal networks for hazard assessment of debris flows. In: Advanced Methods for Decision Making and Risk Management in Sustainability Science, pp. 237-256.

Antonucci, A., Sun, Y., de Campos, C. P., Zaffalon, M., 2010. Generalized loopy 2U: a new algorithm for approximate inference in credal networks. International Journal of Approximate Reasoning, 51(5), 474-484.

Aven, T., Zio, E., 2011. Some considerations on the treatment of uncertainties in risk assessment for practical decision-making. Reliability Engineering and System Safety, 96, 6474.

Azzimonti, L., Corani, G., Zaffalon, M., 2019. Hierarchical estimation of parameters in Bayesian networks. Computational Statistics and Data Analysis, 137, 67-91.

Baudrit, C., Destercke, S., Wuillemin, P.H., 2016. Unifying parameter learning and modelling complex systems with epistemic uncertainty using probability interval. Information Sciences, 367, 630-647.

Bauer, E., Koller, D., Singer, Y., 1997. Update rules for parameter estimation in Bayesian networks. In: Proceedings of the 13th Conference on Uncertainty in Artificial Intelligence, pp. $3-13$.

Benavoli, A., Ristic, B., Farina, A., Oxenham, M., Chisci, L., 2009. An application of evidential networks to threat assessment. IEEE Trans. Aerospace Electron Syst., 45(2), 620-639.

Benferhat, S., Dubois, D., Garcia, L., Prade, H., 2002. On the transformation between possibilistic logic bases and possibilistic causal networks. International Journal of Approximate Reasoning, 29(2), 135-173. 
834 Benjumeda, M., Luengo-Sanchez, S., Larrañaga, P., Bielza, C. 2019. Tractable learning of 835 Bayesian networks from partially observed data. Pattern Recognition, 91, 190-199.

836 Beretta, S., Castelli, M., Gonçalves, I., Henriques, R., Ramazzotti, D., 2018. Learning the 837 structure of Bayesian Networks: A quantitative assessment of the effect of different algorithmic 838 schemes. Complexity, 2018, Article ID 1591878, 12 pages.

839 Beuzen, T., Simmons, J., 2019. A variable selection package driving Netica with Python. 840 Environmental modelling \& software, 115, 1-5.

841 Brooker, P., 2011. Experts, Bayesian belief networks, rare events and aviation risk estimates. 842 Safety Science, 49(8), 1142-1155.

843 Beuzen, T., Marshall, L., Splinter, K.D. 2018. A comparison of methods for discretizing 844 continuous variables in Bayesian Networks. Environmental modelling \& software, 108, 61-66.

845 Bolt, J., van der Gaag, L.C., 2010. An empirical study of the use of the noisy-OR model in a 846 real-life Bayesian network. In: International Conference on Information Processing and 847 Management of Uncertainty in Knowledge-Based Systems. Springer, Berlin, Heidelberg, pp. $848 \quad 11-20$.

849 Cain, J., 2001. Planning Improvements in Natural Resources Management. In: Guidelines for 850 Using Bayesian Networks to Support the Planning and Management of Development 851 Programmes in the Water Sector and Beyond. Centre for Ecology and Hydrology, Wallingford, 852 UK.

853

854

855

856

857

858

859

860

861

862

863

864

865

866

867

868

869

870

871

872

873

de Campos, C.P., Cozman, F.G., 2005. The inferential complexity of Bayesian and credal networks. In: Proceedings of the Nineteenth International Joint Conference on Artificial Intelligence, pp. 1313-1318.

de Campos, C.P., Ji, Q., 2008. Improving Bayesian network parameter learning using constraints, In: Proceedings of the 19th International Joint Conference on Artificial Intelligence, Edinburgh, pp. 1-4.

Cano, A., Gómez, M., Moral, S., Abellán, J., 2007. Hill-climbing and branch-and-bound algorithms for exact and approximate inference in credal networks. International Journal of Approximate Reasoning, 44(3), 261-280.

Castillo, E., Gutiérrez, J.M., Hadi, A.S., 1997. Sensitivity analysis in discrete Bayesian networks. IEEE Transactions on Systems, Man, and Cybernetics, Part A: Systems and Humans, 27(4), 412-423.

Chan, H., Darwiche, A., 2002. When do numbers really matter? J. Artif. Intell. Res., 17, 265287.

Chan, H., Darwiche, A., 2005. A distance measure for bounding probabilistic belief change. International Journal of Approximate Reasoning, 38, 149-174.

Chang, R., Wang, W., 2010. Novel algorithm for Bayesian network parameter learning with informative prior constraints. In: Proceedings of the International Joint Conference on Neural Networks, 2010, pp.1-8.

Chen, S.H., Pollino, C.A., 2012. Good practice in Bayesian network modelling. Environmental modelling \& software, 37, 134-45. 
Chin, K.S., Tang, D.W., Yang, J.B., Wong, S.Y., Wang, H., 2009. Assessing new product development project risk by Bayesian network with a systematic probability generation methodology. Expert Systems with Applications, 36(6), 9879-9890.

877 Chojnacki, E., Plumecocq, W., Audouin, L., 2019. An expert system based on a Bayesian 878 network for fire safety analysis in nuclear area. Fire Safety Journal, 105, 28-40.

879

880

881

882

883

884

885

886

887

888

889

890

891

892

893

894

895

896

897

898

899

900

901

902

903

904

905

906

907

908

909

910

911

912

913

914

915

916

Cooper, G.F., 1984. NESTOR: a Computer-based Medical Diagnostic Aid that Integrates Causal and Probabilistic Knowledge. Report HPP-84-48, Stanford University.

Coupé, V.M.H., van der Gaag, L.C., 2002. Properties of sensitivity analysis of Bayesian belief networks Ann. Math. Artif. Intell., 36, 323-356.

Cowell, R.G., 1999. Parameter learning from incomplete data using maximum entropy I: principles, Statistical Research Report, 21.

Cozman, F.G.. 2000. Credal networks. Artificial Intelligence, 120, 199-233.

Cozman, F.G., 2005. Graphical models for imprecise probabilities. International Journal of Approximate Reasoning 39(2-3), 167-184.

D’Angelo, M.F., Palhares, R.M., Cosme, L.B., Aguiar, L.A., Fonseca, F.S., Caminhas, W.M., 2014. Fault detection in dynamic systems by a fuzzy/Bayesian network formulation. Appl. Soft Comput., 21, 647-653.

Dempster, A.P., 1967. Upper and lower probabilities induced by a multivalued mapping. The annals of mathematical statistics, 325-339.

Dempster, A.P., Laird, N.M., Rubin, D.B., 1977. Maximum likelihood from incomplete data via the EM algorithm. The Royal Statistical Society Series, B39, 1-38.

Dechter, R., 1998. Bucket elimination: A unifying framework for probabilistic inference. In: Learning and Inference in Graphical Models, Springer, Dordrecht, pp. 75-104.

Deng, X., Jiang, W., 2018. Dependence assessment in human reliability analysis using an evidential network approach extended by belief rules and uncertainty measures. Annals of Nuclear Energy, 117, 183-193.

Diez, F.J., 1993. Parameter adjustment in Bayes networks. The generalized noisy or-gate. In Proceedings of the ninth international conference on uncertainty. In: artificial intelligence, Morgan Kaufmann Publishers Inc., USA, pp. 99-105

Ditlevsen, O., 1994. Distribution arbitrariness in structural reliability. In: Proceedings of ICOSSAR'93. Structural Safety \& Reliability, Rotterdam, The Netherlands, pp. 1241-1247.

Drury, B., Valverde-Rebaza, J., Moura, M. F., de Andrade Lopes, A., 2017. A survey of the applications of Bayesian networks in agriculture. Engineering Applications of Artificial Intelligence, 65, 29-42.

Druzdzel, M.J., van der Gaag, L., 2000. Building Probabilistic Networks: "where do the numbers come from?” IEEE Trans. Knowl. Data Eng., 12(4), 481-486.

Dubois, D. Prade, H., 1988. Possibility theory, New York: Plenum.

Dubois, D., Fusco, G., Prade, H., Tettamanzi, A.G., 2017. Uncertain logical gates in possibilistic networks: Theory and application to human geography. International Journal of Approximate Reasoning, 82, 101-118.

Estrada-Lugo, H.D., Patelli, E., de Angelis, M., Raj, D.D., 2018. Bayesian networks with imprecise datasets: application to oscillating water column. In: Safety and Reliability-Safe Societies in a Changing World. CRC Press, pp. 2611-2618. 
917 Fallet-Fidry, G., Weber, P., Simon, C., Iung, B., Duval, C., 2012. Evidential network-based 918 extension of Leaky Noisy-OR structure for supporting risks analyses. In: IFAC Proceedings 919 45(20), 672-677.

920 Feelders, A., van der Gaag, L.C., 2006. Learning Bayesian network parameters under order 921 constraints, International Journal of Approximate Reasoning, 42(1-2), 37-53.

922 Feelders, A., van der Gaag, L.C., 2005. Learning Bayesian network parameters with prior 923 knowledge about context-specific qualitative influences. In: Proceedings of the $31^{\text {st }}$ Conference 924 Annual Conference on Uncertainty in Artificial Intelligence (UAI), pp. 193-200.

925 Feng, G., Zhang, J.D., Liao, S.S., 2014. A novel method for combining Bayesian networks, 926 theoretical analysis, and its applications. Pattern Recognition, 47(5), 2057-2069.

Fenton, N.E., 2018. Handling Uncertain Priors in Basic Bayesian Reasoning. 928 doi10.13140/RG.2.2.16066.89280

930

Fenton, N.E., Noguchi, T., Neil, M., 2019. An extension to the noisy OR function to resolve 931 the 'explaining away' deficiency for practical Bayesian network problems. IEEE Transactions on Knowledge and Data Engineering, in press.

Fenton, N.E., Neil, M., Caballero, J.G., 2007. Using ranked nodes to model qualitative judgments in Bayesian networks IEEE Trans. Knowl. Data Eng., 19(10), 1420-1432.

935

Flage, R., Aven, T., Zio, E., Baraldi, P., 2014. Concerns, challenges, and directions of development for the issue of representing uncertainty in risk assessment. Risk Analysis, 34(7), 1196-1207.

937 Friedberg, I., Hong, X., McLaughlin, K., Smith, P., Miller, P.C. 2017. Evidential network 938 modeling for cyber-physical system state inference. IEEE Access, 5, 17149-17164.

Gao, X.G., Guo, Z.G., Ren, H., Yang, Y., Chen, D.Q., He, C.C., 2019. Learning Bayesian network parameters via minimax algorithm. International Journal of Approximate Reasoning, $941108,62-75$.

Gehl, P., D’Ayala, D., 2016. Development of Bayesian Networks for the multi-hazard fragility assessment of bridge systems. Structural Safety, 60, 37-46.

Geman, S., Geman, D., 1984. Stochastic relaxation, Gibbs distribution and the Bayesian restoration of images, IEEE Transactions on Pattern Analysis and Machine Intelligence, 6, 721946741.

947 Guo, Z.G., Gao, X. G., Ren, H., Yang, Y., Di, R.H., Chen, D.Q., 2017. Learning Bayesian network parameters from small data sets: A further constrained qualitatively maximum a posteriori method. International Journal of Approximate Reasoning, 91, 22-35.

Guth, M., 1991. A probability foundation for vagueness and imprecision in fault tree analysis. IEEE Trans. Reliab., 40(5), 563-570.

952 Hänninen, M., 2014. Bayesian networks for maritime traffic accident prevention: benefits and challenges. Accident Analysis \& Prevention, 73, 305-312.

954 Hänninen, M., Kujala, P., 2012. Influences of variables on ship collision probability in a 955 Bayesian belief network model. Reliability Engineering \& System Safety, 102, 27-40. 

management. Expert Systems with Applications, 41(17), 7837-7846.

He, L., Topa Gomes, A., Broggi, M., Beer, M., 2018. Risk analysis of infinite slope failure using advanced Bayesian networks. In: 8th International Workshop on Reliable Engineering Computing, "Computing with Confidence", 16-18 July 2018, University of Liverpool, Liverpool, UK

Heckerman, D., Geiger, D., Chickering, D.M., 1995. Learning Bayesian networks: the combination of knowledge and statistical data. Machine Learning, 20, 197-243.

Henrion, M., 1989. Some practical issues in constructing belief networks, Uncertainty in Artificial Intelligence 3, Elsevier Sciences Publisher, North Holland.

Henderson, C., Pollino, C.A., Hart, B.T., 2009. Appendix A: the review - workshop discussion document. In: Hart, B.T., Pollino, C.A. (Eds.), Bayesian Modelling for Risk-based Environmental Water Allocation, Waterlines Report Series No. 14, Canberra, pp. 33-83.

Heinze-Deml, C., Maathuis, M.H., Meinshausen, N., 2018. Causal structure learning. Annual Review of Statistics and Its Application, 5, 371-391.

Hooper, P.M., 2008. Exact distribution theory for belief net responses. Bayesian Analysis, 3(3), 615-624.

Hoffman, F.O., Hammonds, J.S., 1994. Propagation of uncertainty in risk assessments: the need to distinguish between uncertainty due to lack of knowledge and uncertainty due to variability. Risk analysis, 14(5), 707-712.

Hospedales, T., Zhou, Y., Fenton, N., Neil, M., 2015. Probabilistic graphical models parameter learning with transferred prior and constraints. In: Proceedings of the 31st Conference on Uncertainty in Artificial Intelligence, AUAI Press, pp. 972-981.

Ide, J.S., Cozman, F.G., 2008. Approximate algorithms for credal networks with binary variables. International Journal of Approximate Reasoning, 48(1), 275-296.

İçen, D., Ersel, D., 2019. A new approach for probability calculation of fuzzy events in Bayesian Networks. International Journal of Approximate Reasoning, 108, 76-88.

Iooss, B., Lemaître, P., 2015. A review on global sensitivity analysis methods. In: Uncertainty management in simulation-optimization of complex systems. Springer, Boston, MA, pp. 101122.

Jaeger, M., 2006. The AI\&M procedure for learning from incomplete data. In: Proceedings of the 22nd Conference on Uncertainty in Artificial Intelligence, pp. 225-232.

Jäger, W.S., Christie, E.K., Hanea, A.M., den Heijer, C., Spencer, T., 2018. A Bayesian network approach for coastal risk analysis and decision making. Coastal Engineering, 134, 48-61.

Janghorbani, A., Moradi, M.H., 2017. Fuzzy evidential network and its application as medical prognosis and diagnosis models. Journal of biomedical informatics, 72, 96-107.

Jensen, F., 2001. Bayesian Networks and Decision Graphs, Springer, New York.

Jøsang, A., 2001. A logic for uncertain probabilities, Int. J. Uncertain. Fuzziness Knowl. Based Syst. 9(3), 279-311. 
Jøsang, A., 2016. Subjective Logic: A Formalism for Reasoning Under Uncertainty, Springer, 996 Heidelberg.

997 Kaplan, L., Ivanovska, M., 2018. Efficient belief propagation in second-order Bayesian 998 networks for singly-connected graphs. International Journal of Approximate Reasoning, 93, $999 \quad 132-152$.

Khakzad, N., 2019. System safety assessment under epistemic uncertainty: Using imprecise probabilities in Bayesian network. Safety Science, 116, 149-160.

1002

Kim, K.J., Cho, S.B., 2017. Ensemble bayesian networks evolved with speciation for highperformance prediction in data mining. Soft Computing, 21(4), 1065-1080.

Kleiter, G.D., 1996. Propagating imprecise probabilities in Bayesian networks. Artificial Intelligence, 88, 143-161.

Klir, G.J., 1989. Is theremore to uncertainty than some probability theorists might have us believe?. International Journal of General System, 15(4), 347-378.

Klir, G.J., 1994. On the alleged superiority of probabilistic representation of uncertainty. IEEE 1009 Transactions on Fuzzy Systems, 2(1), 27-31.

1010

Knochenhauer, M., Swaling, V.H., Dedda, F.D., Hansson, F., Sjökvist, S., Sunnegaerd, K. 2013. Using Bayesian Belief Network (BBN) modelling for rapid source term prediction. Final report (No. NKS--293). Nordisk Kernesikkerhedsforskning, Roskilde, Denmark.

Kuhnert, P.M., Martin, T.G., Griffiths, S.P., 2010. A guide to eliciting and using expert knowledge in Bayesian ecological models. Ecol. Lett., 13, 900-914.

1015 Kullback, S., Leibler, R.A., 1951. On information and sufficiency. The annals of mathematical statistics, 22(1), 79-86.

1017 Kwag, S., Gupta, A., 2017. Probabilistic risk assessment framework for structural systems under multiple hazards using Bayesian statistics. Nuclear Engineering and Design, 315, 20-34.

Laitila, P., Virtanen, K., 2016. Improving construction of conditional probability tables for ranked nodes in Bayesian networks. IEEE Transactions on Knowledge and Data Engineering, 28(7), 1691-1705.

Langseth, H., Portinale, L., 2007. Bayesian networks in reliability. Reliability Engineering and System Safety, 92(1), 92-108.

Laskey, K.B., 1995. Sensitivity analysis for probability assessments in Bayesian networks IEEE

Leonelli, M., 2019. Sensitivity analysis beyond linearity. International Journal of Approximate Reasoning, in press.

1028 Leonelli, M., Goergen, C., Smith, J.Q., 2017. Sensitivity analysis in multilinear probabilistic models. Information Sciences, 411, 84-97. improve the quantification of organizational influences in HRA frameworks. Safety science, 
Li, C., Mahadevan, S., 2018. Sensitivity analysis of a Bayesian network. ASCE-ASME Journal of Risk and Uncertainty in Engineering Systems, Part B: Mechanical Engineering, 4(1), 011003.

Liao, W., Ji, Q., 2009. Learning Bayesian network parameters under incomplete data with domain knowledge, Pattern Recognition, 42(11), 3046-3056.

Loschetter, A., Rohmer, J., de Lary, L., Manceau, J., 2016. Dealing with uncertainty in risk assessments in early stages of a co2 geological storage project: comparison of pure-probabilistic and fuzzy-probabilistic frameworks. Stochastic Environmental Research and Risk Assessment, 30(3), 813-829.

Malagrino, L.S., Roman, N.T., Monteiro, A.M., 2018. Forecasting stock market index daily direction: a Bayesian network approach. Expert Systems with Applications, 105, 11-22.

Marcot, B.G., Steventon, J.D., Sutherland, G.D., McCann, R.K., 2006. Guidelines for developing and updating Bayesian belief networks applied to ecological modeling and conservation. Canadian Journal of Forest Research (Revue Canadienne De Recherche Forestiere), 36, 3063-3074.

Marcot, B.G., Penman, T.D., 2019. Advances in Bayesian network modelling: Integration of modelling technologies. Environmental modelling \& software, 111, 386-393.

Masegosa, A.R., Feelders, A.J., van der Gaag, L.C., 2016. Learning from incomplete data in Bayesian networks with qualitative influences. International Journal of Approximate Reasoning, 69, 18-34.

Mauá, D.D., de Campos, C.P., Zaffalon, M., 2012. Updating credal networks is approximable in polynomial time. International Journal of Approximate Reasoning, 53(8), 1183-1199.

Mi, J., Li, Y. F., Peng, W., Huang, H.Z., 2018. Reliability analysis of complex multi-state system with common cause failure based on evidential networks. Reliability Engineering \& System Safety, 174, 71-81.

Milns, I., Beale, C.M., Smith, V.A., 2010. Revealing ecological networks using Bayesian network inference algorithms. Ecology, 91(7), 1892-1899.

Misuri, A., Khakzad, N., Reniers, G., Cozzani, V., 2018. Tackling uncertainty in security assessment of critical infrastructures: Dempster-Shafer Theory vs. Credal Sets Theory. Safety science, 107, 62-76.

Mkrtchyan, L., Podofillini, L., Dang, V.N., 2015. Bayesian belief networks for human reliability analysis: A review of applications and gaps. Reliability engineering \& system safety, 139, 1-16.

Musharraf, M., Bradbury-Squires, D., Khan, F., Veitch, B., MacKinnon, S., Imtiaz, S., 2014. A virtual experimental technique for data collection for a Bayesian network approach to human reliability analysis. Reliability Engineering \& System Safety, 132, 1-8.

Neil, M., Tailor, M., Marquez, D., 2007. Inference in hybrid Bayesian networks using dynamic discretization. Statistics and Computing, 17(3), 219-233.

Neil, M., Fenton, N., Nielson, L., 2000. Building large-scale Bayesian networks. The Knowledge Engineering Review, 15(3), 257-284. 
1073

1074

1075

1076

1077

1078

1079

1080

1081

1082

1083

1084

1085

1086

1087

1088

1089

1090

1091

1092

1093

1094

1095

1096

1097

1098

1099

1100

1101

1102

1103

1104

1105

1106

1107

1108

1109

Norsys Software Corp., 2006. Sensitivity to Findings Netica Software Documentation available at www.norsys.com

Niculescu, R.S., Mitchell, T.M., Rao, R.B., 2006. Bayesian network learning with parameter constraints, J. Mach. Learn. Res., 7(3), 1357-1383.

Nojavan, F., Qian, S.S., Stow, C.A., 2017. Comparative analysis of discretization methods in Bayesian networks. Environmental modelling \& software, 87, 64-71.

Oniśko, A., Druzdzel, M. J., Wasyluk, H., 2001. Learning Bayesian network parameters from small data sets: Application of Noisy-OR gates. International Journal of Approximate Reasoning, 27(2), 165-182.

Pearl, J., 1988. Probabilistic Reasoning in Intelligent Systems. Morgan Kaufmann, San Francisco, CA

Pedroni, N., Zio, E., Ferrario, E., Pasanisi, A., Couplet, M., 2013. Hierarchical propagation of probabilistic and non-probabilistic uncertainty in the parameters of a risk model. Computers \& Structures, 126, 199-213.

Penz, C.A., Flesch, C.A., Nassar, S.M., Flesch, R.C.C., De Oliveira, M.A., 2012. Fuzzy Bayesian network for refrigeration compressor performance prediction and test time reduction, Expert Systems with Applications, 39, 4268-4273.

Podofillini, L, Mkrtchyan, L., Dang, V.N., 2014. Aggregating expert-elicited error probabilities to build HRA models. In: Proceedings of ESREL 2014. Sept. 14-18, Wroclaw, Poland; 2014, pp. 1083-1091.

Pollard, E., Rombaut, M., Pannetier, B., 2010. Bayesian networks vs. evidential networks: An application to convoy detection. In: International Conference on Information Processing and Management of Uncertainty in Knowledge-Based Systems. Springer, Berlin, Heidelberg, pp. 31-39.

Ramoni, M., Sebastiani, P., 2001. Robust learning with missing data. Machine Learning, 45(2), 147-170.

Ren, J., Jenkinson, I., Wang, J., Xu, D., Yang, J., 2009. An offshore risk analysis method using fuzzy Bayesian network. J. Offshore Mech. Arct. Eng., 131, 041101.

Renooij, S., 2001. Probability elicitation for belief networks: issues to consider. Knowled. Eng. Rev., 16, 255-269.

Renooij, S., 2014. Co-variation for sensitivity analysis in bayesian networks: properties, consequences and alternatives. International Journal of Approximate Reasoning, 55, 10221042 .

Røed, W., Mosleh, A., Vinnem, J.E., Aven, T., 2009. On the use of the hybrid causal logic method in offshore risk analysis. Reliability Engineering and System Safety, 94(2), 445-455.

Scutari, M., Howell, P., Balding, D. J., Mackay, I., 2014. Multiple quantitative trait analysis using Bayesian networks. Genetics, 198(1), 129-137. 
Scutari, M., Graafland, C.E., Gutierrez, J.M., 2018. Who Learns Better Bayesian Network Structures:Constraint-Based, Score-based or Hybrid Algorithms? In: Proceedings of Machine Learning Research, 72, pp. 1-12.

Seixas, F.L., Zadrozny, B., Laks, J., Conci, A., Saade, D.C.M., 2014. A Bayesian network decision model for supporting the diagnosis of dementia, Alzheimer's disease and mild cognitive impairment. Comput. Biol. Med., 51, 140-158.

Shafer, G., 1976. A Mathematical Theory of Evidence. Princeton University Press, Princeton, NJ.

Simon, C., Bicking, F., 2017. Hybrid computation of uncertainty in reliability analysis with pbox and evidential networks. Reliability Engineering \& System Safety, 167, 629-638.

Simon, C., Weber, P., 2009. Evidential networks for reliability analysis and performance evaluation of systems with imprecise knowledge. IEEE Transactions on Reliability, 58(1), 6987.

Simon, C., Weber, P., Evsukoff, A., 2008. Bayesian networks inference algorithm to implement Dempster Shafer theory in reliability analysis. Reliability Engineering and System Safety, 93(7), 950-963.

Smets, P., 2002. The application of the matrix calculus to belief functions. International Journal of Approximate Reasoning, 31, 1-30.

Tang, H., Liu, S., 2007. Basic theory of fuzzy Bayesian networks and its application in machinery fault diagnosis. In: Proceedings of Fourth International Conference on Fuzzy Systems and Knowledge Discovery, 24-27 Aug. 2007, Haikou, Hainan, China, pp.132-137.

Thiesson, B., 1995 Accelerated quantification of Bayesian networks with incomplete data, In: Proceedings of the First International Conference on Knowledge Discovery and Data Mining, pp. 306-311.

Tolo, S., Patelli, E., Beer, M., 2017. Robust vulnerability analysis of nuclear facilities subject to external hazards. Stochastic Environmental Research and Risk Assessment, 31(10), 27332756.

Tolo, S., Patelli, E., Beer, M., 2018. An open toolbox for the reduction, inference computation and sensitivity analysis of Credal Networks. Advances in Engineering Software, 115, 126-148.

Van Allen, T., Singh, A., Greiner, R.Hooper, P.M., 2008. Quantifying the uncertainty of a belief net response: Bayesian error-bars for belief net inference. Artificial Intelligence, 172, 483-513.

Van der Gaag, L.C., Kuijper, R., Van Geffen, Y.M., Vermeulen, J.L., 2013. Towards uncertainty analysis of Bayesian Networks. In 25th Benelux Conference on Artificial Intelligence, Delft, The Netherlands.

Verbert, K., Babuška, R., De Schutter, B., 2017. Bayesian and Dempster-Shafer reasoning for knowledge-based fault diagnosis-A comparative study. Engineering Applications of Artificial Intelligence, 60, 136-150.

Walley, P. 1991. Statistical reasoning with imprecise probabilities. Chapman and Hall, London. 
Weber, P., Medina-Oliva, G., Simon, C., Iung, B., 2012. Overview on Bayesian networks applications for dependability, risk analysis and maintenance areas. Engineering Applications of Artificial Intelligence, 25(4), 671-682.

Werner, C., Bedford, T., Cooke, R.M., Hanea, A.M., Morales-Napoles, O., 2017. Expert judgement for dependence in probabilistic modelling: a systematic literature review and future research directions. European Journal of Operational Research, 258(3), 801-819.

Wiegmann, D.A., 2005. Developing a methodology for eliciting subjective probability estimates during expert evaluations of safety interventions: application for bayesian belief networks. Technical report from Aviation Human Factors Division AH FD-05-13/NASA-054. https://ntrs.nasa.gov/archive/nasa/casi.ntrs.nasa.gov/20050238445.pdf

Wisse, B.W., van Gosliga, S.P., van Elst, N.P., Barros, A.I., 2008. Relieving the elicitation burden of bayesian belief networks. In: Proceedings of the sixth Bayesian modelling applications workshop on UAI. Helsinki, Finland.

Woudenberg, S.P., van der Gaag, L.C., 2015. Propagation effects of model-calculated probability values in Bayesian networks. International Journal of Approximate Reasoning, 61, $1-15$.

Xu, H., Smets, P., 1996. Reasoning in evidential networks with conditional belief functions. International Journal of Approximate Reasoning, 14(2-3), 155-185.

Yang, Y., Gao, X., Guo, Z., Chen, D., 2019. Learning Bayesian networks using the constrained maximum a posteriori probability method. Pattern Recognition, 91, 123-134.

Yang, J., Huang, H. Z., Liu, Y., Li, Y.F., 2012. Evidential networks for fault tree analysis with imprecise knowledge. Int. J. Turbo Jet Engines, 29(2), 111-122.

Zadeh, L.A., 1965. Fuzzy sets. Information and control, 8(3), 338-353.

Zadeh, L.A., 1975. The concept of a linguistic variable and its application to approximate reasoning. Information sciences, 8(3), 199-249.

Zagorecki, A., Druzdzel, M.J., 2004. An empirical study of probability elicitation under noisyor assumption. In: Flairs conference, pp. 880-886.

Zagorecki, A., Druzdzel, M.J., 2012. Knowledge engineering for Bayesian networks: How common are noisy-MAX distributions in practice?. IEEE Transactions on Systems, Man, and Cybernetics: Systems, 43(1), 186-195.

Zhang, X., Mahadevan, S., Deng, X., 2017. Reliability analysis with linguistic data: An evidential network approach. Reliability Engineering \& System Safety, 162, 111-121.

Zhang, G., Thai, V.V., 2018. Addressing the epistemic uncertainty in maritime accidents modelling using Bayesian network with interval probabilities. Safety science, 102, 211-225.

Zhang, L., Wu, X., Qin, Y., Skibniewski, M.J., Liu, W., 2015. Towards a fuzzy Bayesian network based approach for safety risk analysis of tunnel-induced pipeline damage, Risk Anal. 36(2), 278-301. 
1185 Zhou, Y., Fenton, N., Neil, M., 2014. Bayesian network approach to multinomial param- eter 1186 learning using data and expert judgments. International Journal of Approximate Reasoning, 1187 55(5), 1252-1268.

1188 Zhou, Y., Fenton, N., Zhu, C., 2016a. An empirical study of Bayesian network parameter 1189 learning with monotonic influence constraints. Decis. Support Syst., 87(C), 69-79 .

1190 Zhou, K., Martin, A., Pan, Q., 2016b. The belief noisy-or model applied to network reliability 1191 analysis. International Journal of Uncertainty, Fuzziness and Knowledge-Based Systems, 1192 24(6), 937-960.

1193 Zhang, G., Thai, V.V., 2016. Expert elicitation and Bayesian Network modeling for shipping 1194 accidents: A literature review. Safety science, 87, 53-62.

1195 Zwirglmaier, K., Straub, D., 2016. A discretization procedure for rare events in Bayesian 1196 networks. Reliability Engineering \& System Safety, 153, 96-109. 